\title{
LARGE DEVIATION PRINCIPLES FOR NON-UNIFORMLY HYPERBOLIC RATIONAL MAPS
}

\author{
HENRI COMMAN $^{\dagger}$ AND JUAN RIVERA-LETELIER ${ }^{\ddagger}$
}

\begin{abstract}
We show some level-2 large deviation principles for rational maps satisfying a strong form of non-uniform hyperbolicity, called "Topological Collet-Eckmann". More precisely, we prove a large deviation principle for the distribution of iterated preimages, periodic points, and Birkhoff averages. For this purpose we show that each Hölder continuous potential admits a unique equilibrium state, and that the pressure function can be characterized in terms of iterated preimages, periodic points, and Birkhoff averages. Then we use a variant of a general result of Kifer.
\end{abstract}

\section{INTRODUCTION}

This paper is devoted to the study of (level-2) large deviation principles for complex rational maps of degree at least two, viewed as dynamical systems acting on the Riemann sphere. Our results apply to rational maps satisfying a strong form of non-uniform hyperbolicity condition, called "Topological Collet-Eckmann" (TCE). Although the TCE condition is very strong, the set of rational maps that satisfy it, but that are not uniformly hyperbolic, has positive Lebesgue measure in the space of rational maps of a given degree [Asp04]; see also [Ree86, GŚ00, Smi00, DF08] for related results. The TCE condition is also interesting because it can be formulated in several equivalent ways PRLS03.

The first key observation is that for a rational map satisfying the TCE condition every Hölder continuous potential has a unique equilibrium state. This allows us to apply (a variant of) a general result of Kifer Kif90, Theorem 3.4] to obtain level-2 large deviation principles for sequences of measures associated to periodic points, iterated preimages, and Birkhoff averages.

We now proceed to describe our results in more detail.

2000 Mathematics Subject Classification. Primary: 37D35; Secondary: 37A50, 37D25, $60 \mathrm{~F} 10$.

Key words and phrases. Large deviation principle, thermodynamic formalism, rational map, non-uniform hyperbolicity, Topological Collet-Eckmann condition.

$\dagger$ Partially supported by FONDECYT grant 1070045. Gratefully acknowledges Universidad Católica del Norte for hospitality.

$\ddagger$ Partially supported by Research Network on Low Dimensional Systems, PBCT/CONICYT, Chile. Gratefully acknowledges Universidad de Santiago de Chile for hospitality. 
1.1. Equilibrium states for TCE rational maps. Let $T$ be a complex rational map of degree at least two, viewed as a dynamical system acting on the Riemann sphere $\overline{\mathbb{C}}$. We denote by $J(T)$ its Julia set and by $\mathscr{M}(J(T), T)$ the space of invariant probability measures supported by $J(T)$, endowed with the weak* topology. For each $\mu \in \mathscr{M}(J(T), T)$ we denote by $h_{\mu}(T)$ the measure-theoretic entropy of $\mu$. Given a Hölder continuous function $\varphi: J(T) \rightarrow \mathbb{R}$, a probability measure $\mu_{0} \in \mathscr{M}(J(T), T)$ is called an equilibrium state of $T$ for the potential $\varphi$, if the supremum

$$
P(T, \varphi):=\sup \left\{h_{\mu}(T)+\int \varphi d \mu: \mu \in \mathscr{M}(J(T), T)\right\}
$$

is attained at $\mu=\mu_{0}$.

The TCE condition was originally formulated in topological terms. It is equivalent to the following strong form of Pesin's non-uniform hyperbolicity condition: There is a constant $\chi>0$ such that for each $\mu \in \mathscr{M}(J(T), T)$ the Lyapunov exponent $\int \log \left|T^{\prime}\right| d \mu$ of $\mu$ is greater than or equal to $\chi$. See PRLS03] for the original formulation of the TCE condition, and several others equivalent formulations. For other results concerning equilibrium states of rational maps see MS03, PRL08, SU03 and references therein.

The following result is fundamental in what follows.

Theorem A. Let $T$ be a rational map satisfying the TCE condition. Then for every Hölder continuous function $\varphi: J(T) \rightarrow \mathbb{R}$ there is a unique equilibrium state of $T$ for the potential $\varphi$.

We obtain this theorem as a simple consequence of [Dob08, Theorem 8]. In Appendix $\mathrm{A}$ we give a reasonably self contained proof of this result, as a consequence of a Ruelle-Perron-Frobenius type theorem (Theorem D). When the potential $\varphi$ satisfies $\sup _{J(T)} \varphi<P(T, \varphi)$, these results were shown for a general rational map $T$ in [DU91a, Prz90, DPU96]. The fact that Theorem A holds for every Hölder continuous potential is crucial to obtain the large deviation principles that we proceed to describe.

1.2. Level-2 large deviations principles for TCE rational maps. Let $\mathscr{M}(J(T))$ be the space of Borel probability measures on $J(T)$ endowed with the weak* topology, and let $I: \mathscr{M}(J(T)) \rightarrow[0,+\infty]$ be a lower semicontinuous function. Recall that a sequence $\left(\Omega_{n}\right)_{n \geq 1}$ of Borel probability measures on $\mathscr{M}(J(T))$ is said to satisfy a large deviation principle with rate function $I$, if for every closed subset $\mathscr{F}$ of $\mathscr{M}(J(T))$ we have

$$
\limsup _{n \rightarrow+\infty} \frac{1}{n} \log \Omega_{n}(\mathscr{F}) \leq-\inf _{\mathscr{F}} I
$$

and if for every open subset $\mathscr{G}$ of $\mathscr{M}(J(T))$ we have,

$$
\liminf _{n \rightarrow+\infty} \frac{1}{n} \log \Omega_{n}(\mathscr{G}) \geq-\inf _{\mathscr{G}} I .
$$

The function $I$ is uniquely characterized by this property, see $\oint 2$ for background and further properties. 
Theorem B. Let $T$ be a rational map satisfying the TCE condition, let $\varphi$ : $J(T) \rightarrow \mathbb{R}$ be a Hölder continuous function, and let $\mu_{\varphi}$ be the unique equilibrium state of $T$ for the potential $\varphi$. For each integer $n \geq 1$ let $W_{n}: J(T) \rightarrow \mathscr{M}(J(T))$ be the continuous function defined by

$$
W_{n}(x):=\frac{1}{n}\left(\delta_{x}+\delta_{T(x)}+\cdots+\delta_{T^{n-1}(x)}\right),
$$

and let $S_{n}(\varphi): J(T) \rightarrow \mathbb{R}$ be defined by

$$
S_{n}(\varphi)(x):=n \int \varphi d W_{n}(x)=\varphi(x)+\varphi \circ T(x)+\cdots+\varphi \circ T^{n-1}(x) .
$$

Given an integer $n \geq 1$ consider the following Borel probability measures on $\mathscr{M}(J(T))$.

Periodic points: Letting $\operatorname{Per}_{n}:=\left\{p \in J(T) \mid T^{n}(p)=p\right\}$, put

$$
\Omega_{n}:=\sum_{p \in \operatorname{Per}_{n}} \frac{\exp \left(S_{n}(\varphi)(p)\right)}{\sum_{p^{\prime} \in \operatorname{Per}_{n}} \exp \left(S_{n}(\varphi)\left(p^{\prime}\right)\right)} \delta_{W_{n}(p)} .
$$

Iterated preimages: Given $x_{0} \in J(T)$, put

$$
\Omega_{n}\left(x_{0}\right):=\sum_{x \in T^{-n}\left(x_{0}\right)} \frac{\exp \left(S_{n}(\varphi)(x)\right)}{\sum_{y \in T^{-n}\left(x_{0}\right)} \exp \left(S_{n}(\varphi)(y)\right)} \delta_{W_{n}(x)} .
$$

Birkhoff averages: $\Sigma_{n}:=W_{n}\left[\mu_{\varphi}\right]$ (i.e., the image measure of $\mu_{\varphi}$ by $\left.W_{n}\right)$.

Then each of the sequences $\left(\Omega_{n}\right)_{n \geq 1},\left(\Omega_{n}\left(x_{0}\right)\right)_{n \geq 1}$ and $\left(\Sigma_{n}\right)_{n \geq 1}$ converges to $\delta_{\mu_{\varphi}}$ in the weak* topology, and satisfies a large deviation principle in $\mathscr{M}(J(T))$ with rate function $I^{\varphi}: \mathscr{M}(J(T)) \rightarrow[0,+\infty]$ given by

$$
I^{\varphi}(\mu)= \begin{cases}P(T, \varphi)-\int \varphi d \mu-h_{\mu}(T) & \text { if } \mu \in \mathscr{M}(J(T), T) ; \\ +\infty & \text { if } \mu \in \mathscr{M}(J(T)) \backslash \mathscr{M}(J(T), T) .\end{cases}
$$

Furthermore, for each convex open subset $\mathscr{G}$ of $\mathscr{M}(J(T))$ containing some invariant measure we have $\inf _{\mathscr{G}} I^{\varphi}=\inf _{\bar{G}} I^{\varphi}$, and

$$
\lim _{n \rightarrow+\infty} \frac{1}{n} \log \Omega_{n}(\mathscr{G})=\lim _{n \rightarrow+\infty} \frac{1}{n} \log \Omega_{n}\left(x_{0}\right)(\mathscr{G})=\lim _{n \rightarrow+\infty} \frac{1}{n} \log \Sigma_{n}(\mathscr{G})=\inf _{\mathscr{G}} I^{\varphi},
$$

and the above expression remains true replacing $\mathscr{G}$ by $\overline{\mathscr{G}}$.

In order to illustrate Theorem B we state a couple of corollaries.

Corollary 1.1. Let $\psi: J(T) \rightarrow \mathbb{R}$ be a continuous function, and let $\widehat{\psi}: \mathscr{M}(J(T)) \rightarrow \mathbb{R}$ be defined by $\widehat{\psi}(\mu)=\int \psi d \mu$. With the notations of Theorem $\mathbb{B}$, each of the sequences of image measures $\left(\widehat{\psi}\left[\Omega_{n}\right]\right)_{n \geq 1},\left(\widehat{\psi}\left[\Omega_{n}\left(x_{0}\right)\right]\right)_{n \geq 1}$, $\left(\widehat{\psi}\left[\Sigma_{n}\right]\right)_{n \geq 1}$ satisfies a large deviation principle in $\mathbb{R}$ with rate function

$$
x \mapsto \inf \left\{I^{\varphi}(\mu): \mu \in \mathscr{M}(J(T)), \int \psi d \mu=x\right\} .
$$


Furthermore, when $\psi$ is normalized so that $\int \psi d \mu_{\varphi}=0$, for each $\varepsilon>0$ small enough we have

$$
\begin{aligned}
& \lim _{n \rightarrow+\infty} \frac{1}{n} \log \left(\frac{\sum_{p \in \operatorname{Per}_{n}, \frac{1}{n}\left|S_{n}(\psi)(p)\right|>\varepsilon} \exp \left(S_{n}(\varphi)(p)\right)}{\sum_{p^{\prime} \in \mathrm{Per}_{n}} \exp \left(S_{n}(\varphi)\left(p^{\prime}\right)\right)}\right) \\
= & \lim _{n \rightarrow+\infty} \frac{1}{n} \log \left(\frac{\sum_{x \in T^{-n}\left(x_{0}\right), \frac{1}{n}\left|S_{n}(\psi)(x)\right|>\varepsilon} \exp \left(S_{n}(\varphi)(x)\right)}{\sum_{y \in T^{-n}\left(x_{0}\right)} \exp \left(S_{n}(\varphi)(y)\right)}\right) \\
= & \lim _{n \rightarrow+\infty} \frac{1}{n} \log \mu_{\varphi}\left\{x \in J(T): \frac{1}{n}\left|S_{n}(\psi)(x)\right|>\varepsilon\right\}
\end{aligned}
$$

$$
=-\inf \left\{P(T, \varphi)-\int \varphi d \mu-h_{\mu}(T): \mu \in \mathscr{M}(J(T), T),\left|\int \psi d \mu\right|>\varepsilon\right\},
$$

and the above limits are strictly negative (possibly infinite).

Corollary 1.2. With the notations of Theorem $\mathbb{B}$, for each $\mu \in \mathscr{M}(J(T), T)$ and each convex local basis $\mathscr{G}_{\mu}$ at $\mu$, we have

$$
\begin{aligned}
h_{\mu}(T) & +\int \varphi d \mu \\
& =\inf \left\{\lim _{n \rightarrow+\infty} \frac{1}{n} \log \sum_{p \in \operatorname{Per}_{n}, W_{n}(p) \in \mathscr{G}} \exp \left(S_{n}(\varphi)(p): \mathscr{G} \in \mathscr{G}_{\mu}\right\},\right. \\
& =\inf \left\{\lim _{n \rightarrow+\infty} \frac{1}{n} \log \sum_{x \in T^{-n}\left(x_{0}\right), W_{n}(x) \in \mathscr{G}} \exp \left(S_{n}(\varphi)(x): \mathscr{G}_{\in} \in \mathscr{G}_{\mu}\right\},\right. \\
& =P(T, \varphi)+\inf \left\{\lim _{n \rightarrow+\infty} \frac{1}{n} \log \mu_{\varphi}\left\{x \in J(T): W_{n}(x) \in \mathscr{G}\right\}: \mathscr{G} \in \mathscr{G}_{\mu}\right\} .
\end{aligned}
$$

Theorem B was obtained recently by the first named author in the case where $T$ is uniformly hyperbolic Com09, Theorem 2] 1 For the same class of maps, the case of Birkhoff averages and $\varphi=0$ was obtained earlier by Lopes [Lop90, and the upper-bounds in the case of periodic points were proved by Pollicott and Sridharan in [PS07].

The Birkhoff averages case of Theorem $\mathrm{B}$ was obtained by Grigull when $T$ is a parabolic rational map and when the potential $\varphi$ satisfies $\sup _{\overline{\mathbb{C}}} \varphi<$ $P(T, \varphi)$ [Gri93, Theorem 1]. See also the survey paper of Denker [Den96].

The large deviation upper bounds in the case of iterated preimages have been proved by Pollicott and Sharp in PS96] for an arbitrary rational

\footnotetext{
${ }^{1}$ Taking $T$ uniformly hyperbolic in Theorem $\mathrm{B}$ does not permit to recover all the cases treated in Com09, Theorem 2]; this comes from the fact that in this last paper, the potential $\varphi$ need not have a unique equilibrium state, as it is required in Theorem B (see Com09, Example 4.1]).
} 
map $T$, when the potential $\varphi$ satisfies $\sup _{J(T)} \varphi<P(T, \varphi)$. An alternative proof of this result can be obtained using a general result on upper bounds, see [Com09, Remark 2 and Theorem 4] and [DZ98, Theorem 4.5.3]. See also PSY98 for the upper bounds in the case of interval maps with indifferent periodic points.

Using the contraction principle it is possible to derive from Theorem B a level-1 large deviation principle in $\mathbb{R}$ for each continuous potential, as in Corollary 1.1]. However, this simple trick does not work with the geometric potential $-\log \left|T^{\prime}\right|$ by the lack of continuity of the evaluation map $\mu \mapsto \int \log \left|T^{\prime}\right| d \mu$ when there is a critical point in the Julia set. The techniques needed in order to get (even partial) level-1 large deviations with the potential $-\log \left|T^{\prime}\right|$ are different from those used here, and we shall not tackle them in this paper. We refer here to results where large deviation bounds are proved only for some subsets of the real line, like for example those obtained by Keller and Nowicki [KN92, Theorem 1.2 and Theorem 1.3] in the case of unimodal maps satisfying the Collet-Eckmann condition, or [PRL08, Corollary B.4] and [XF07] in the case of rational maps 3 .

1.3. Abstract result on level-2 large deviations principles. Theorem $\mathrm{B}$ is obtained as a particular case of the following variant of Kifer's result [Kif90, Theorem 3.4]. See Appendix B for an extension to more general dynamical systems and nets in place of sequences.

Theorem C. Let $X$ be a compact metrizable topological space, and let $T$ : $X \rightarrow X$ be a continuous map such that the measure-theoretic entropy of $T$, as a function defined on $\mathscr{M}(X, T)$, is finite and upper semi-continuous. Fix $\varphi \in C(X)$, and let $\mathcal{W}$ be a dense vector subspace of $C(X)$ such that for each $\psi \in \mathcal{W}$ there is a unique equilibrium state of $T$ for the potential $\varphi+\psi$. Let $I^{\varphi}: \mathscr{M}(X) \rightarrow[0,+\infty]$ be the function defined by

$$
I^{\varphi}(\mu)= \begin{cases}P(T, \varphi)-\int \varphi d \mu-h_{\mu}(T) & \text { if } \mu \in \mathscr{M}(X, T) \\ +\infty & \text { if } \mu \in \mathscr{M}(X) \backslash \mathscr{M}(X, T) .\end{cases}
$$

Then every sequence $\left(\Omega_{n}\right)_{n \geq 1}$ of Borel probability measures on $\mathscr{M}(X)$ such that for every $\psi \in \mathcal{W}$,

$$
\lim _{n \rightarrow+\infty} \frac{1}{n} \log \int_{\mathscr{M}(X)} \exp \left(n \int \psi d \mu\right) d \Omega_{n}(\mu)=P(T, \varphi+\psi)-P(T, \varphi),
$$

satisfies a large deviation principle with rate function $I^{\varphi}$, and it converges in the weak* topology to the Dirac mass supported on the unique equilibrium state of $T$ for the potential $\varphi$. Furthermore, for each convex and open subset $\mathscr{G}$ of $\mathscr{M}(X)$ containing some invariant measure, we have

$$
\lim _{n \rightarrow+\infty} \frac{1}{n} \log \Omega_{n}(\mathscr{G})=\lim _{n \rightarrow+\infty} \frac{1}{n} \log \Omega_{n}(\overline{\mathscr{G}})=-\inf _{\mathscr{G}} I^{\varphi}=-\inf _{\overline{\mathscr{G}}} I^{\varphi} .
$$

\footnotetext{
${ }^{2}$ See also the inducing scheme approach (of level-1 large deviations) given recently by Melbourne and Nicol MN08, and Rey-Bellet and Young RBY08.

${ }^{3}$ See [DS08] for a weak form of upper bounds in the higher dimensional setting.
} 
The method we use to prove Theorem $\mathrm{C}$ is in line with the general functional approach of large deviations in probability theory. This approach seems to have been initiated by Sievers in Sie69 and then by Plachky and Steinebach in Pla71, PS75 in order to generalize to sequences of dependent random variables the large deviation principle proved by Cramer (in a special case) and Chernoff (in the general case) for the laws of empirical means of independent and identically distributed random variables in $\mathbb{R}$ Cra38, Che52. The result was extended to $\mathbb{R}^{d}$-valued random variables in Gär77, and then refined by Ellis leading to the well-known Gärtner-Ellis theorem in [Ell84], that was later generalized by Baldi in [Bal88] to real topological vector spaces.

For the case of dynamical systems, Takahashi in [Tak84, Tak87] studied the large deviation functional associated to the distributions of Birkhoff averages with respect to some (not necessarily invariant) measure. Then, in a very general setting, Kifer gave sufficient conditions in order to get the large deviation principle with convex rate function, for empirical measures Kif90, Theorem 2.1]. This result can be seen as a purely theoretic large deviation one, in the sense that the hypotheses do not depend on a system under which the empirical measures could evolve (see Remark 3.3). This allowed Kifer to derive more specific results for dynamical systems; the first one concerns the distribution of these empirical measures with respect to some reference measure, like in the third case of Theorem B Kif90, Theorem 3.4] (see Appendix B); the second one deals with the case where these measures are governed by a Markov process [Kif90, Theorem 4.1]. Recently, the first named author gave another type of sufficient condition in order to get a large deviation principle with the same rate function [Com09, Theorem 4].

In all the above results, the first basic assumption relates the pressure to the large deviation functional associated to the sequence or net of measures (see \$2). Roughly speaking, it is required that the (translated) pressure functional coincides with the large deviation functional; rigorously, this means that (1.5) holds for all $\psi \in C(X)$ (or equivalently, for all $\psi$ in a dense subset of $C(X))$. It turns out that the existence of the limit in the left hand side of (1.5) is also necessary in order to have the large deviation principle, and the fact that it coincides with the pressure is necessary in order to have the rate function of Theorem B (see Remark 3.2).

The second basic assumption is in fact a condition on the large deviation functional in disguise; we refer the reader to Remark 3.3 and Appendix B in the case of Kifer's theorem. In the case of [Com09], it is required that every invariant measure can be approximated in the weak* topology, and in entropy, by measures which are unique equilibrium states for some potentials; when (1.5) holds for all $\psi \in C(X)$, this turns out to be the usual Baldi's condition in large deviation theory [Bal88].

We can summarize the functional approach by saying it consists to look for sufficient conditions on the large deviation functional implying the large deviation principle. The rate function (1.2) is then a natural candidate when 
the first above mentioned basic assumption holds, since in this case it is the only possible convex rate function (namely, the Legendre-Fenchel transform of the restriction of the large deviation functional to the topological dual of the space of finite signed Borel measures on $X$ (i.e. $C(X)$ ); see DZ98] and Com09] in connection with Remark 3.3).

1.4. Organization. After some preliminaries in 92 , we give the proof of Theorem $\mathrm{C}$ in 93 . In Appendix $\mathrm{B}$ we use this result to give another variant of Kifer's result for semi-flows [Kif90, Theorem 3.4], that we state as Theorem E

We start 4 by deriving the proof of Theorem A from Dob08, Theorem 8] in 4.1. Then we obtain Theorem $\mathrm{B}$ and its corollaries in 4.3 , from Theorem $\mathrm{A}$ and Theorem $\mathrm{C}$, using several characterizations of the pressure given in 84.2 .

In Appendix A we give a reasonably self contained proof of Theorem A as a consequence of a Ruelle-Perron-Frobenius type theorem (Theorem D).

1.5. Acknowledgements. We thank Godofredo Iommi for a useful remark concerning Theorem $\mathrm{A}$.

\section{Preliminaries}

2.1. Notation. We denote by $\overline{\mathbb{R}}=\mathbb{R} \cup\{-\infty,+\infty\}$ the extended real line. We denote by dist the spherical metric on $\overline{\mathbb{C}}$. Given a subset $E$ of $\overline{\mathbb{C}}$ we denote by $\mathbf{1}_{E}$ the indicator function of $E$. We will denote $\mathbf{1}_{\overline{\mathbb{C}}}$ simply by $\mathbf{1}$.

2.2. Measure spaces. Given a compact metric space $X$, we denote by $C(X)$ the space of continuous functions defined on $X$ taking images in $\mathbb{R}$, endowed with the uniform topology. We identify the dual of $C(X)$ with the space $\widetilde{\mathscr{M}}(X)$ of finite signed Borel measures on $X$ endowed with the weak* topology [DS88, $§ I V .6$, Theorem 3]. We denote by $\mathscr{M}(X) \subset \widetilde{\mathscr{M}}(X)$ the space of Borel probability measures on $X$, and recall that $\mathscr{M}(X)$ is compact [DS88, §V.4, Theorem 2] and metrizable [DS88, §V.5, Theorem 1]. If $T: X \rightarrow X$ is a continuous map, then we denote by $\mathscr{M}(X, T)$ the compact subset of $\mathscr{M}(X)$ constituted by the measures that are invariant by $T$.

2.3. Convex analysis. Let $\mathcal{X}$ be a locally convex Hausdorff real topological vector space, and let $\mathcal{X}^{*}$ be its topological dual. The Legendre-Fenchel transform of a function $f: \mathcal{X} \rightarrow \overline{\mathbb{R}}$ is by definition the function $f^{*}: \mathcal{X}^{*} \rightarrow \overline{\mathbb{R}}$ defined by

$$
f^{*}(u)=\sup \{u(x)-f(x): x \in \mathcal{X}\} .
$$

The duality theorem asserts that if $f$ is convex, lower semi-continuous and takes values in $(-\infty,+\infty]$, then for each $x \in \mathcal{X}$ we have

$$
f(x)=\sup \left\{u(x)-f^{*}(u): u \in \mathcal{X}^{*}\right\}
$$

see for example [ET76, §I, Proposition 4.1]. 
2.4. Thermodynamic formalism. The reader may refer to Wal82, Rue04 for background in ergodic theory and thermodynamic formalism, and [PU02, Zin96 for an introduction in the case of rational maps.

Let $X$ be a compact metric space with metric $d$, and let $T: X \rightarrow X$ be a continuous map. For $\mu \in \mathscr{M}(X, T)$ we will denote by $h_{\mu}(T)$ the measuretheoretic entropy of $\mu$. We now recall the definition of topological pressure through " $(n, \varepsilon)$-separated sets", that will be needed in 84.2 . Denote by $T \times T: X \times X \rightarrow X \times X$ the diagonal action defined by $T \times T\left(x, x^{\prime}\right)=$ $\left(T(x), T\left(x^{\prime}\right)\right)$. Given an integer $n \geq 1$ we denote by $d_{n}$ the distance on $X$ defined by

$$
d_{n}=\max \left\{d \circ(T \times T)^{j}: j \in\{0, \ldots, n-1\}\right\} .
$$

Note that $d_{1}=d$. Given $\varepsilon>0$ we say that a subset $\mathscr{N}$ of $X$ is $(n, \varepsilon)$-separated, if for each pair of distinct elements $x, x^{\prime}$ of $\mathscr{N}$ we have $d_{n}\left(x, x^{\prime}\right)>\varepsilon$. For an integer $n \geq 1$ and a continuous function $\varphi: X \rightarrow \mathbb{R}$ we put

$$
S_{n}(\varphi)=\varphi+\varphi \circ T+\cdots+\varphi \circ T^{n-1} .
$$

Then the pressure function is equal to

$$
P(T, \varphi)=\lim _{\varepsilon \rightarrow 0} \lim _{n \rightarrow+\infty} \sup _{\mathscr{N}} \sum_{y \in \mathscr{N}} \exp \left(S_{n}(\varphi)(y)\right),
$$

where the supremum is taken over all $(n, \varepsilon)$-separated subsets $\mathscr{N}$ of $X$. The fact that the pressure function defined with $(n, \varepsilon)$-separated sets as above is equal to the supremum in (1.1), is known as the variational principle. When the topological entropy of $T$ is finite, the topological pressure viewed as a function defined on $C(X)$, takes finite values and it is Lipschitz continuous [Wal82, Theorem 9.7].

2.5. Large deviations. We recall here some basic facts of large deviation theory that will be used in the sequel. Since we will allude to large deviations for nets in place of sequences, and in various types of topological spaces, we state them in a general topological setting, and refer the reader to [DZ98, Com03, Com07, Ell85, for more details.

Let $\left(\Omega_{\alpha}\right)$ be a net of Borel probability measures on a Hausdorff topological space $\mathscr{X}$, and let $\left(t_{\alpha}\right)$ be a net in $(0,+\infty)$ converging to 0 . We say that $\left(\Omega_{\alpha}\right)$ satisfies a large deviation principle with powers $\left(t_{\alpha}\right)$ if there exists a lower semi-continuous function $I: \mathscr{X} \rightarrow[0,+\infty]$ such that

$$
\limsup _{t_{\alpha} \rightarrow 0} t_{\alpha} \log \Omega_{\alpha}(\mathscr{F}) \leq-\inf \{I(x): x \in \mathscr{F}\} \quad \text { for all closed } \mathscr{F} \subset \mathscr{X},
$$

and

$$
\liminf _{t_{\alpha} \rightarrow 0} t_{\alpha} \log \Omega_{\alpha}(\mathscr{G}) \geq-\inf \{I(x): x \in \mathscr{G}\} \quad \text { for all open } \mathscr{G} \subset \mathscr{X} .
$$


Such a function $I$ is then unique when $\mathscr{X}$ is regular; it is called the rate function, and is given for each $x \in \mathscr{X}$ and each local basis $\mathscr{G}_{x}$ at $x$ by

$$
\begin{aligned}
-I(x) & =\inf \left\{\liminf _{t_{\alpha} \rightarrow 0} t_{\alpha} \log \Omega_{\alpha}(\mathscr{G}): \mathscr{G} \in \mathscr{G}_{x}\right\} \\
& =\inf \left\{\limsup _{t_{\alpha} \rightarrow 0} t_{\alpha} \log \Omega_{\alpha}(\mathscr{G}): \mathscr{G} \in \mathscr{G}_{x}\right\} .
\end{aligned}
$$

A Borel set $\mathscr{A} \subset \mathscr{X}$ is called a $I$-continuity set if

$$
\inf \{I(x): x \in \operatorname{Interior}(\mathscr{A})\}=\inf \{I(x): x \in \overline{\mathscr{A}}\} .
$$

When (2.1) and (2.2) hold, then $\lim _{t_{\alpha} \rightarrow 0} t_{\alpha} \log \Omega_{\alpha}(\mathscr{A})$ exists and satisfies

$$
\lim _{t_{\alpha} \rightarrow 0} t_{\alpha} \log \Omega_{\alpha}(\mathscr{A})=-\inf \{I(x): x \in \mathscr{A}\}
$$

and we can replace $\mathscr{A}$ by either its interior or its closure in the above equality. When only (2.1) (resp. (2.2)) is satisfied, we say that the large deviation upper (resp. lower) bounds hold with the function $I$.

The contraction principle asserts that when $\left(\Omega_{\alpha}\right)$ is supported by a compact subset of $\mathscr{X}$ and $\left(\Omega_{\alpha}\right)$ satisfies a large deviation principle with powers $\left(t_{\alpha}\right)$ and rate function $I$, then for every Hausdorff topological space $\mathscr{Y}$ and any continuous map $g: \mathscr{X} \rightarrow \mathscr{Y}$ the net of image measures $\left(g\left[\Omega_{\alpha}\right]\right)$ satisfies a large deviation principle with powers $\left(t_{\alpha}\right)$ and rate function defined on $\mathscr{Y}$ by

$$
y \mapsto \inf \{I(x): x \in \mathscr{X}, g(x)=y\} .
$$

The large deviation functional associated to $\left(\Omega_{\alpha}\right)$ and $\left(t_{\alpha}\right)$ is the map defined on the set of $[-\infty,+\infty)$-valued Borel functions $h$ on $\mathscr{X}$ by

$$
h \mapsto \limsup _{t_{\alpha} \rightarrow 0} t_{\alpha} \log \int \exp \left(h / t_{\alpha}\right) d \Omega_{\alpha} ;
$$

it is continuous with respect to the uniform metric. Assume that $\mathscr{X}$ is a Hausdorff real topological vector space, let $\mathscr{X}^{*}$ denote its topological dual endowed with the weak* topology, and let $\bar{L}$ be the restriction of the large deviation functional (2.4) to $\mathscr{X}^{*}$; for $u \in \mathscr{X}^{*}$ we shall write $L(u)$ when the limit exists in (2.4). When the net $\left(\Omega_{\alpha}\right)$ is supported by a compact subset $\mathscr{K} \subset \mathscr{X}$, then $\bar{L}$ is a convex lower semi-continuous function. In the literature, $\bar{L}$ is also known as the "generalized log-moment generating function", "free-energy", or "pressure", depending of the context.

The above notions will be applied with $\mathscr{X}=\widetilde{\mathscr{M}}(X)$ (strictly speaking, $\mathscr{X}$ will be homeomorphic to $\widetilde{\mathscr{M}}(X)), \mathscr{K}=\mathscr{M}(X), \mathscr{Y}=\mathbb{R}$ and $g=\widehat{\psi}$ for some $\psi \in C(X)$, where $\widehat{\psi}$ is the evaluation map (i.e. $\left.\widehat{\psi}(\mu)=\int \psi d \mu\right)$. Note that if $L(\widehat{\psi})$ exists for all $\psi$ in a dense subset of $C(X)$, then $L(\widehat{\psi})$ exists for all $\psi \in C(X)$. In this context, the large deviation principles in $\mathscr{M}(X)$, or more generally in $\widetilde{\mathscr{M}}(X)$, are usually referred to as "level-2", and the ones in $\mathbb{R}$ (in particular those obtained by contraction) as "level-1". 


\section{Proof of Theorem C}

This section is devoted to the proof of Theorem C. It is based on Lemma 3.1 below, which identifies the rate function as a Legendre-Fenchel transform. Throughout the rest of this section we fix $X, T, \mathcal{W}, \varphi$ as in the statement of Theorem C. Note that the hypothesis of Theorem $\mathrm{C}$, that the measure-theoretic entropy is finite and upper semi-continuous, implies that for every $\psi \in C(X)$ the pressure $P(T, \psi)$ is finite.

Lemma 3.1. Let $Q_{\varphi}: C(X) \rightarrow \mathbb{R}$ be the function defined by

$$
Q_{\varphi}(\psi)=P(T, \varphi+\psi)-P(T, \varphi) .
$$

Then the following properties hold.

1. The function $Q_{\varphi}$ is continuous, convex, and its Legendre-Fenchel transform $Q_{\varphi}^{*}$ is given by

$$
Q_{\varphi}^{*}(\mu)= \begin{cases}P(T, \varphi)-\int \varphi d \mu-h_{\mu}(T) & \text { if } \mu \in \mathscr{M}(X, T) \\ +\infty & \text { if } \mu \in \widetilde{\mathscr{M}}(X) \backslash \mathscr{M}(X, T) .\end{cases}
$$

In particular $Q_{\varphi}^{*}$ takes images in $[0,+\infty]$, and it vanishes precisely on the set of equilibrium states of $T$ for the potential $\varphi$. Note furthermore that $Q_{\varphi \mid \mathscr{M}(X)}^{*}=I^{\varphi}$.

2. For each $\psi \in C(X)$, a measure $\mu \in \mathscr{M}(X, T)$ is an equilibrium state of $T$ for the potential $\varphi+\psi$ if and only if $Q_{\varphi}(\psi)=\int \psi d \mu-Q_{\varphi}^{*}(\mu)$.

Proof. The convexity and the continuity of $Q_{\varphi}$ follow from the same properties of the pressure function, see 92.4 . Let $U: \widetilde{\mathscr{M}}(X) \rightarrow \overline{\mathbb{R}}$ be defined by

$$
U(\mu)= \begin{cases}-\int \varphi d \mu-h_{\mu}(T) & \text { if } \mu \in \mathscr{M}(X, T) \\ +\infty & \text { if } \mu \in \widetilde{\mathscr{M}}(X) \backslash \mathscr{M}(X, T) .\end{cases}
$$

Since the measure-theoretic entropy of $T$ is affine, and since it is upper semicontinuous by hypothesis, it follows that the function $U$ is convex, lower semi-continuous, and that it takes values in $(-\infty,+\infty]$. By the variational principle, for each $\psi \in C(X)$ we have

$$
\begin{aligned}
P(T, \varphi+\psi) & =\sup \left\{h_{\mu}(T)+\int \varphi+\psi d \mu: \mu \in \mathscr{M}(X, T)\right\} \\
& =\sup \left\{\int \psi d \mu-U(\mu): \mu \in \widetilde{\mathscr{M}}(X)\right\} .
\end{aligned}
$$

This shows that the function $\psi \mapsto P(T, \varphi+\psi)$ is the Legendre-Fenchel transform of $U$. Hence, the duality theorem implies that for each $\mu \in \widetilde{\mathscr{M}}(X)$ 
we have,

$$
\begin{aligned}
U(\mu) & =\sup \left\{\int \psi d \mu-P(T, \varphi+\psi): \psi \in C(X)\right\} \\
& =\sup \left\{\int \psi d \mu-P(T, \varphi)-Q_{\varphi}(\psi): \psi \in C(X)\right\} \\
& =-P(T, \varphi)+\sup \left\{\int \psi d \mu-Q_{\varphi}(\psi): \psi \in C(X)\right\} \\
& =-P(T, \varphi)+Q_{\varphi}{ }^{*}(\mu) .
\end{aligned}
$$

This proves part 1 . Then part 2 follows from the equalities

$$
\begin{aligned}
P(T, \varphi+\psi)- & h_{\mu}(T)-\int(\varphi+\psi) d \mu \\
=Q_{\varphi}(\psi)+P(T, \varphi)-h_{\mu}(T) & -\int(\varphi+\psi) d \mu \\
& =Q_{\varphi}(\psi)+Q_{\varphi}{ }^{*}(\mu)-\int \psi d \mu .
\end{aligned}
$$

Proof of Theorem $\square$, Let $\mathcal{X}$ be the space of all linear functionals on $\mathcal{W}$ endowed with the $\mathcal{W}$-topology, i.e. the coarsest topology such that for each $\psi \in \mathcal{W}$ the evaluation map $\widehat{\psi}: \mathcal{X} \rightarrow \mathbb{R}$ defined by $\widehat{\psi}(u)=u(\psi)$ is continuous. Note that $\mathcal{X}$ is a locally convex real topological vector space with topological dual $\mathcal{X}^{*}=\{\widehat{\psi} \mid \psi \in \mathcal{W}\}$. Given $\mu \in \mathscr{M}(X)$ denote by $\pi(\mu)$ the element of $\mathcal{X}$ such that for each $\psi \in \mathcal{W}$ we have $\pi(\mu)(\psi)=\int \psi d \mu$, and let $\mathscr{M}_{\mathcal{W}}(X)$ denote the image of the function $\pi: \mathscr{M}(X) \rightarrow \mathcal{X}$ so defined. Since by hypothesis $\mathcal{W}$ is a dense subspace of $C(X)$, the map $\pi$ is an homeomorphism from $\mathscr{M}(X)$ onto $\mathscr{M}_{\mathcal{W}}(X)$; in particular, $\mathscr{M}_{\mathcal{W}}(X)$ is a compact subset of $\mathcal{X}$. We shall prove the large deviation principle for the sequence $\left(\pi\left[\Omega_{n}\right]\right)_{n \geq 1}$ in $\mathscr{M}_{\mathcal{W}}(X)$, and the corresponding statement for $\left(\Omega_{n}\right)_{n \geq 1}$ in $\mathscr{M}(X)$ will follow from the fact that $\pi$ is a homeomorphism. Let $\bar{L}$ be the restriction to $\mathcal{X}^{*}$ of the large deviation functional associated to $\left(\pi\left[\Omega_{n}\right]\right)_{n \geq 1}$, seen as a sequence of measures on $\mathcal{X}$; recall that for $\psi \in \mathcal{W}$ for which the limsup defining $\bar{L}(\widehat{\psi})$ is a limit, we denote $\bar{L}(\widehat{\psi})$ by $L(\widehat{\psi})$ (see $\S 2.5)$. By (1.5) we have for each $\psi \in \mathcal{W}$

$$
L(\widehat{\psi})=\lim _{n \rightarrow+\infty} \frac{1}{n} \log \int_{\mathcal{X}} \exp (n \widehat{\psi}) d \pi\left[\Omega_{n}\right]=Q_{\varphi}(\psi) .
$$

Since $\mathcal{W}$ is dense in $C(X)$ and $Q_{\varphi}$ is continuous (Lemma 3.1), we get for each $\mu \in \mathscr{M}(X)$,

$$
L^{*}(\pi(\mu))=Q_{\varphi}^{*}(\mu) .
$$

The hypotheses on $\mathcal{W}$ imply that $Q_{\varphi}$ is Gateaux differentiable at each $\psi \in \mathcal{W}$ by part 2 of Lemma 3.1 ET99, Proposition 5.3], which by (3.1) is equivalent to the Gateaux differentiability of $L$ on $\mathcal{X}^{*}$. It follows that all the hypotheses 
of [DZ98, Corollary 4.6.14] applied to the sequence $\left(\pi\left[\Omega_{n}\right]\right)_{n \geq 1}$ are verified, and consequently $\left(\pi\left[\Omega_{n}\right]\right)_{n \geq 1}$ satisfies a large deviation principle in $\mathcal{X}$ with rate function $L^{*}$. Since $\mathscr{M}_{\mathcal{W}}(X)$ is closed in $\mathcal{X}$ the large deviation principle holds in $\mathscr{M}_{\mathcal{W}}(X)$ with rate function $\left.L^{*}\right|_{\mathscr{M}_{\mathcal{W}}(X)}$ [DZ98, Lemma 4.1.5], and thus with rate function $Q_{\varphi}^{*} \circ \pi^{-1}=I^{\varphi} \circ \pi^{-1}$ by (3.2).

To prove that $\left(\Omega_{n}\right)_{n \geq 1}$ converges to the Dirac mass at the unique equilibrium state $\mu_{\varphi}$ of $T$ for the potential $\varphi$, let $\mathscr{G}$ be an open neighborhood of $\mu_{\varphi}$ in $\mathscr{M}(X)$. Since $I^{\varphi}$ is lower semi-continuous, non-negative, and it vanishes precisely on $\left\{\mu_{\varphi}\right\}$ (Lemma 3.1), the infimum of $I^{\varphi}$ on $\mathscr{F}:=\mathscr{M}(X) \backslash \mathscr{G}$ is attained at some point of $\mathscr{F}$, and thus inf $\mathscr{F} I^{\varphi}>0$. Therefore we have

$$
\limsup _{n \rightarrow+\infty} \frac{1}{n} \log \Omega_{n}(\mathscr{F}) \leq-\inf _{\mathscr{F}} I^{\varphi}<0,
$$

and $\lim _{n \rightarrow+\infty} \Omega_{n}(\mathscr{G})=1$.

To prove the last statement of the theorem, let $\mathscr{G} \subset \mathscr{M}(X)$ be a convex and open set containing an invariant measure $\mu^{\prime}$. Since the function $I^{\varphi}$ is lower semi-continuous, and since it takes finite values precisely on the compact set $\mathscr{M}(X, T)$ (Lemma 3.1), there exists $\mu \in \overline{\mathscr{G}} \cap \mathscr{M}(X, T)$ such that $I^{\varphi}(\mu)=\inf _{\bar{G}} I^{\varphi}$. For each $t \in(0,1)$ put $\mu_{t}=(1-t) \mu+t \mu^{\prime}$, and note that $\mu_{t} \in \mathscr{M}(X, T)$ and $\mu_{t} \in \mathscr{G}$ [Sch71, 1.1, p. 38]. Since the function $I^{\varphi}$ is affine on $\mathscr{M}(X, T)$, we have

$$
\inf _{\mathscr{G}} I^{\varphi} \leq \lim _{t \rightarrow 0} I^{\varphi}\left(\mu_{t}\right)=I^{\varphi}(\mu)=\inf _{\bar{G}} I^{\varphi} .
$$

This shows that $\inf _{\mathscr{G}} I^{\varphi}=\inf _{\bar{G}} I^{\varphi}$. That is, $\mathscr{G}$ is a $I^{\varphi}$-continuity set and the last assertion of the theorem follows (see 92 ).

Remark 3.2. The equality (1.5) is in fact necessary in order to have the large deviation principle with rate function $I^{\varphi}$. Indeed, when such a large deviation principle holds, Varadhan's theorem (DZ98, Theorem 4.3.1], Com03, Corollary 3.4]) states that for each $\psi \in C(X)$ the limit

$$
\lim _{n \rightarrow+\infty} \frac{1}{n} \log \int_{\mathscr{M}(X)} \exp \left(n \int \psi d \mu\right) d \Omega_{n}(\mu)
$$

exists and satisfies

$$
\begin{aligned}
& \lim _{n \rightarrow+\infty} \frac{1}{n} \log \int_{\mathscr{M}(X)} \exp \left(n \int \psi d \mu\right) d \Omega_{n}(\mu) \\
&=\sup \left\{\int \psi d \mu-I^{\varphi}(\mu): \mu \in \mathscr{M}(X)\right\} \\
&=\sup \left\{\int \psi d \mu-Q_{\varphi}{ }^{*}(\mu): \mu \in \widetilde{\mathscr{M}}(X)\right\}=Q_{\varphi}(\psi) .
\end{aligned}
$$

The upper semi-continuity of the measure-theoretic entropy is also necessary since by definition the rate function is lower semi-continuous. 
Remark 3.3. The part of the proof of Theorem C concerning the large deviation principle is a generalization of [Kif90, Theorem 2.1]; indeed, let us consider a sequence of measures $\left(\Omega_{n}\right)_{n \geq 1}$ on $\mathscr{M}(X)$ whose associated limiting large deviation functional $L(\hat{*})$ exists on $\widetilde{\mathscr{M}}(X)^{*}$, and for $\psi \in C(X)$ put $Q(\psi)=L(\widehat{\psi})$. If we assume that $Q$ is Gateaux differentiable at each point of a dense vector subspace $\mathcal{W}$ of $C(X)$ (which is the hypothesis of [Kif90, Theorem 2.1], and the one of Theorem $\mathrm{C}$ with $\left.Q=Q_{\varphi}\right)$, then the proof works verbatim (the convexity of $L$ gives the convexity of $Q$, and the uniform continuity of $L$ gives the continuity of $Q\left(\operatorname{since} \sup _{\mathscr{M}(X)} \widehat{\psi}=\sup _{X} \psi\right)$; the rate function is $Q^{*}$ ). We have used [DZ98, Corollary 4.6.14] instead of [Kif90, Theorem 2.1], first because it is not clear how the proof of [Kif90, Theorem 2.1], which deals with special nets of measures, extends to general sequences, and second because it emphasizes the role of large deviation theory. In fact, DZ98, Corollary 4.6.14] can be thought of as an extension to general locally convex spaces of [Kif90, Theorem 2.1]. Indeed, the latter result deals with $\widetilde{\mathscr{M}}(X)$, which can be identified (via the map $\pi$, and thanks to the fact that $\mathcal{W}$ is dense in $C(X)$ ) with a subspace of the locally convex space $\mathcal{X}$. The hypotheses of Kif90, Theorem 2.1] amount to both the equility (3.1) and the Gateaux differentiability of $L$ on $\mathcal{X}^{*}$, which are precisely the hypotheses of [DZ98, Corollary 4.6.14].

\section{LARGE DEVIATION PRINCIPLES FOR TCE RATIONAL MAPS}

The purpose of this section is to prove Theorem $\mathrm{A}$, as well as Theorem B and its corollaries. The proof of Theorem $\mathrm{A}$ is deduced from Dob08, Theorem 8$]$ in $\$ 4.1$, after recalling some well known definitions and results about transfer operators. After giving several equivalent characterizations of the pressure function in 4.2 , we give the proof of Theorem $\mathrm{B}$ and its corollaries in $\$ 4.3$.

4.1. The transfer operator and conformal measures. Fix a rational map $T: \overline{\mathbb{C}} \rightarrow \overline{\mathbb{C}}$ of degree at least two. For $y \in \overline{\mathbb{C}}$ we denote by $\operatorname{deg}_{T}(y)$ the local degree of $T$ at $y$. Given a continuous function $\varphi: J(T) \rightarrow \mathbb{R}$ we denote by $\mathscr{L}_{\varphi}$ the (Ruelle-Perron-Frobenius) transfer operator, acting on the space of functions defined on $J(T)$ and taking values in $\mathbb{R}$, by

$$
\mathscr{L}_{\varphi}(\psi)(x)=\sum_{y \in T^{-1}(x)} \operatorname{deg}_{T}(y) \exp (\varphi(y)) \psi(y) .
$$

Note that $\mathscr{L}_{\varphi}$ acts continuously on the space of continuous functions. We denote by $\mathscr{L}_{\varphi}^{*}$ the continuous operator acting on $\widetilde{\mathscr{M}}(J(T))$ by

$$
\int \psi d \mathscr{L}_{\varphi}^{*}(\eta)=\int \mathscr{L}_{\varphi}(\psi) d \eta
$$

Note that it maps non-zero measures to non-zero measures. By the change of variable formula it follows that for every Borel measure $\eta$ and every 
measurable function $\psi: J(T) \rightarrow \mathbb{R}$ satisfying $\int|\psi| d \eta<+\infty$, we have $\int\left|\mathscr{L}_{\varphi}(\psi)\right| d \eta<+\infty$ and $\int \psi d \mathscr{L}_{\varphi}^{*}(\eta)=\int \mathscr{L}_{\varphi}(\psi) d \eta$.

Given a continuous function $g: J(T) \rightarrow[0,+\infty)$ we say that a Borel measure $\eta$ supported on $J(T)$ is g-conformal for $T$ if for every subset $E$ of $J(T)$ on which $T$ is injective we have $\eta(T(E))=\int_{E} g d \eta$.

The following lemma is well-known. Part 2 is a special case of DU91b, Proposition 2.2].

Lemma 4.1. Let $T$ be a complex rational map, and let $\varphi: J(T) \rightarrow \mathbb{R}$ be a continuous function. Then the following conclusions hold.

1. There is $\lambda>0$ and a Borel probability measure $\eta$ such that $\mathscr{L}_{\varphi}^{*}(\eta)=$ $\lambda \eta$.

2. For a given $\lambda>0$, a Borel measure $\eta$ supported on $J(T)$ is $\lambda \exp (-\varphi)$-conformal for $T$ if and only if $\mathscr{L}_{\varphi}^{*} \eta=\lambda \eta$.

Proof. Let $\widehat{\mathscr{L}_{\varphi}^{*}}$ be the map acting on $\mathscr{M}(J(T))$ defined by

$$
\widehat{\mathscr{L}}_{\varphi}^{*}(\eta)=\left(\mathscr{L}_{\varphi}^{*}(\eta)(J(T))\right)^{-1} \mathscr{L}_{\varphi}^{*}(\eta) .
$$

As $\widehat{\mathscr{L}}_{\varphi}^{*}$ is continuous and $\mathscr{M}(J(T))$ is compact and convex, the SchauderTychonoff theorem [DS88, $\S \mathrm{V} .10$, Theorem 5] then implies that $\widehat{\mathscr{L}}_{\varphi}^{*}$ has a fixed point $\eta$. Letting $\lambda=\mathscr{L}_{\varphi}^{*}(\eta)(J(T))>0$, we have $\mathscr{L}_{\varphi}^{*}(\eta)=\lambda \eta$.

Note that for every Borel probability measure $\eta$ and every Borel subset $E$ of $J(T)$ on which $T$ is injective, we have $\mathscr{L}_{\varphi}\left(\mathbf{1}_{E} \exp (-\varphi)\right)=\mathbf{1}_{T(E)}$ and,

$$
\begin{aligned}
& \eta(T(E))=\int \mathbf{1}_{T(E)} d \eta \\
&=\int \mathscr{L}_{\varphi}\left(\mathbf{1}_{E} \exp (-\varphi)\right) d \eta=\int \mathbf{1}_{E} \exp (-\varphi) d \mathscr{L}_{\varphi}^{*}(\eta) .
\end{aligned}
$$

So, if for some $\lambda>0$ the measure $\eta$ satisfies $\mathscr{L}_{\varphi}^{*}(\eta)=\lambda \eta$, then $\eta$ is $\lambda \exp (-\varphi)$-conformal. Suppose on the other hand that $\eta$ is $\lambda \exp (-\varphi)$-conformal. Then (4.1) implies that for every Borel subset $E$ of $J(T)$ on which $T$ is injective we have

$$
\int \mathbf{1}_{E} \exp (-\varphi) d \mathscr{L}_{\varphi}^{*} \eta=\lambda \int \mathbf{1}_{E} \exp (-\varphi) d \eta
$$

As $J(T)$ can be partitioned into a finite number of Borel sets on which $T$ is injective, this equality holds in fact for every Borel subset $E$ of $J(T)$. We thus have $\mathscr{L}_{\varphi}^{*} \eta=\lambda \eta$.

Proof of Theorem $\underline{A}$. Let $T$ be a rational map satisfying the TCE condition and let $\varphi: J(T) \rightarrow \mathbb{R}$ be a Hölder continuous function. Since the measuretheoretic entropy of $T$ is upper semi-continuous [FLM83, Lju83, it follows that there is an equilibrium state $\rho$ of $T$ for the potential $\varphi$. To prove the uniqueness, first observe that by Lemma 4.1 there is a $(\exp (P(T, \varphi)-\varphi))$-conformal probability measure for $T$. On the other hand, by [PRLS03, Main Theorem] the Lyapunov exponent of every invariant measure supported on $J(T)$ 
is positive, so [Dob08, Theorem 8] implies that $\rho$ is the unique equilibrium state of $T$ for the potential $\varphi$.

4.2. Characterizations of the pressure function. Given a rational map $T$ satisfying the TCE condition and a Hölder continuous function $\varphi: J(T) \rightarrow$ $\mathbb{R}$, in this section we characterize the pressure function $P(T, \varphi)$ in terms of iterated preimages (Lemma 4.2), periodic points (Lemma 4.3), and Birkhoff averages (Lemma 4.4); compare with PRLS04]. Lemma 4.2 is also used in Appendix A, Compare Lemma 4.4 with Lop90, Theorem 3].

We will make use of the following equivalent formulation of the TCE condition [PRLS03, Main Theorem], for a rational map $T$ of degree at least two.

Exponential Shrinking of Components (ESC). There exist $\lambda_{\mathrm{ESC}}>1$ and $r_{0}>0$ such that for every $x \in J(T)$, every integer $n \geq 1$ and every connected component $W$ of $T^{-n}\left(B\left(x, r_{0}\right)\right)$ we have

$$
\operatorname{diam}(W) \leq \lambda_{\mathrm{ESC}}^{-n} .
$$

Recall that for each integer $n \geq 1$, and each $\psi: J(T) \rightarrow \mathbb{R}$ we denote

$$
S_{n}(\psi)=\psi+\psi \circ T+\cdots+\psi \circ T^{n-1} .
$$

Lemma 4.2. Let $T$ be a rational map satisfying the TCE condition and let $\varphi: J(T) \rightarrow \mathbb{R}$ be a Hölder continuous function. Then there is a constant $C_{0}>0$ such that for each $x \in J(T)$ we have

$$
C_{0}^{-1} \leq \exp (-n P(T, \varphi)) \cdot \mathscr{L}_{\varphi}^{n}(\mathbf{1})(x) \leq C_{0} .
$$

In particular,

$$
\lim _{n \rightarrow+\infty} \frac{1}{n} \log \mathscr{L}_{\varphi}^{n}(\mathbf{1})(x)=P(T, \varphi) .
$$

Proof. Let $\lambda_{\mathrm{ESC}}>1$ and $r_{0}>0$ be as in the ESC condition. Let $\kappa \in$ $(0,1)$ be the exponent of $\varphi$. We will use the following fact several times: If $x, x^{\prime} \in J(T)$ belong to a ball $B$ of radius less than or equal to $r_{0}$ centered at $J(T)$, then for every $y \in T^{-n}(x)$ and $y^{\prime} \in T^{-n}\left(x^{\prime}\right)$ in the same connected component of $T^{-n}(B)$, we have

$$
\left|S_{n}(\varphi)(y)-S_{n}(\varphi)\left(y^{\prime}\right)\right| \leq|\varphi|_{\kappa}\left(2 r_{0}\right)^{\kappa}\left(\lambda_{\mathrm{ESC}}^{\kappa}-1\right)^{-1} .
$$

So, if we put $C_{1}:=\exp \left(|\varphi|_{\kappa}\left(2 r_{0}\right)^{\kappa}\left(\lambda_{\mathrm{ESC}}^{\kappa}-1\right)^{-1}\right)$, then we have

$$
C_{1}^{-1} \leq \mathscr{L}_{\varphi}^{n}(\mathbf{1})(x) / \mathscr{L}_{\varphi}^{n}(\mathbf{1})\left(x^{\prime}\right) \leq C_{1} .
$$

Let $\mathscr{U}$ be a finite covering of $J(T)$ by balls of radius $r_{0}$ centered at $J(T)$.

1. We will show that there is a constant $C_{0}>1$ so that for every integer $n \geq$ 1 , and every $x, x^{\prime} \in J(T)$ we have

$$
C_{0}^{-1} \leq \mathscr{L}_{\varphi}^{n}(\mathbf{1})(x) / \mathscr{L}_{\varphi}^{n}(\mathbf{1})\left(x^{\prime}\right) \leq C_{0} .
$$


By the locally eventually onto property of $T$ on $J(T)$, there is a positive integer $n_{0}$ such that for every $B \in \mathscr{U}$ we have $J(T) \subset T^{n_{0}}(B)$. We will show that for each $n \geq n_{0}$ and $x \in J(T)$ we have

$$
\begin{aligned}
C_{1}^{-1}\left(\sup _{J(T)} \mathscr{L}_{\varphi}^{n-n_{0}}(\mathbf{1})\right) & \left(\inf _{J(T)} \exp (\varphi)\right)^{n_{0}} \leq \mathscr{L}_{\varphi}^{n}(\mathbf{1})(x) \\
& \leq \operatorname{deg}(T)^{n_{0}}\left(\sup _{J(T)} \mathscr{L}_{\varphi}^{n-n_{0}}(\mathbf{1})\right)\left(\sup _{J(T)} \exp (\varphi)\right)^{n_{0}} .
\end{aligned}
$$

The desired assertion follows easily from these inequalities.

The second inequality is an easy consequence of the formula,

$$
\mathscr{L}_{\varphi}^{n}(\mathbf{1})(x)=\sum_{y \in T^{-n_{0}}(x)} \operatorname{deg}_{T^{n_{0}}}(y) \exp \left(S_{n_{0}}(\varphi)(y)\right) \mathscr{L}_{\varphi}^{n-n_{0}}(\mathbf{1})(y) .
$$

and from the fact that $\#\left(T^{-n_{0}}(x)\right) \leq \operatorname{deg}(T)^{n_{0}}$. To prove the first inequality, let $y_{0} \in J(T)$ be such that

$$
\mathscr{L}_{\varphi}^{n-n_{0}}(\mathbf{1})\left(y_{0}\right)=\sup _{J(T)} \mathscr{L}_{\varphi}^{n-n_{0}}(\mathbf{1}) .
$$

Furthermore, let $B \in \mathscr{U}$ containing $y_{0}$, and let $y \in B$ be such that $T^{n_{0}}(y)=$ $x$. Then we have

$$
\mathscr{L}_{\varphi}^{n}(\mathbf{1})(x) \geq \exp \left(S_{n_{0}}(\varphi)(y)\right) \mathscr{L}_{\varphi}^{n-n_{0}}(\mathbf{1})(y) \geq\left(\inf _{J(T)} \exp (\varphi)\right)^{n_{0}} C_{1}^{-1} \mathscr{L}_{\varphi}^{n-n_{0}}(\mathbf{1})\left(y_{0}\right) .
$$

2. We will prove that for each $x \in J(T)$ we have

$$
\lim _{n \rightarrow+\infty} \frac{1}{n} \log \mathscr{L}_{\varphi}^{n} \mathbf{1}(x)=P(T, \varphi) .
$$

Given $\delta>0$ let $\varepsilon>0$ and $n_{0} \geq 1$ be such that for each $n \geq n_{0}$ there is a $(n, \varepsilon)$-separated set $\mathscr{N}$ such that

$$
\sum_{y \in \mathscr{N}} \exp \left(S_{n}(\varphi(y))\right) \geq \exp (n(P(T, \varphi)-\delta)) .
$$

Taking $n_{0}$ larger if necessary, we assume that $\lambda_{\mathrm{ESC}}^{n_{0}} \leq \varepsilon$.

Fix $n \geq n_{0}$, let $\mathscr{N}$ be as above, and let $B \in \mathscr{U}$ be such that the set $\mathscr{N}_{B}:=\left\{y \in \mathscr{N} \mid T^{n+n_{0}}(y) \in B\right\}$ satisfies

$$
\sum_{y \in \mathscr{N}_{B}} \exp \left(S_{n}(\varphi)(y)\right) \geq \frac{1}{\# \mathscr{U}} \exp (n(P(T, \varphi)-\delta)) .
$$

Since for each $m=n_{0}, n_{0}+1, \ldots, n$ the diameter of each connected component of $T^{-m}(B)$ is less than or equal to $\lambda_{\text {ESC }}^{m} \leq \varepsilon$, it follows that each connected component of $T^{-\left(n+n_{0}\right)}(B)$ can contain at most one element of $\mathscr{N}$. 
Therefore for each $x \in B \cap J(T)$ we have

$$
\begin{aligned}
\mathscr{L}_{\varphi}^{n+n_{0}}(\mathbf{1})(x) \geq C_{1}^{-1} & \left(\inf _{J(T)} \exp (\varphi)\right)^{n_{0}} \sum_{y \in \mathscr{N}_{B}} \exp \left(S_{n}(\varphi)(y)\right) \\
& \geq C_{1}^{-1}\left(\inf _{J(T)} \exp (\varphi)\right)^{n_{0}} \frac{1}{\# \mathscr{U}} \exp (n(P(T, \varphi)-\delta)) .
\end{aligned}
$$

Together with part 1 this implies that for each $x^{\prime} \in J(T)$ we have

$$
\liminf _{n \rightarrow+\infty} \frac{1}{n} \log \mathscr{L}_{\varphi}^{n}(\mathbf{1})\left(x^{\prime}\right) \geq P(T, \varphi)-\delta .
$$

Since $\delta>0$ was arbitrary, this shows that for each $x^{\prime} \in J(T)$ we have

$$
\liminf _{n \rightarrow+\infty} \frac{1}{n} \log \mathscr{L}_{\varphi}^{n}(\mathbf{1})\left(x^{\prime}\right) \geq P(T, \varphi) .
$$

It remains to prove that for each $x \in J(T)$ we have,

$$
\limsup _{n \rightarrow+\infty} \frac{1}{n} \log \mathscr{L}_{\varphi}^{n}(\mathbf{1})(x) \leq P(T, \varphi) .
$$

Let $\varepsilon>0$ be given. For each $n \geq 1$ and $y_{0} \in J(T)$ denote by $N_{n}\left(y_{0}\right)$ the number of points in $T^{-n}\left(T^{n}\left(y_{0}\right)\right)$, counted with multiplicity, that are $(n, \varepsilon)$-close to $y_{0}$, and put $N_{n}:=\sup _{y_{0} \in J(T)} N_{n}\left(y_{0}\right)$. Then, for every $n \geq 1$ and $x_{0} \in J(T)$ the set $T^{-n}\left(x_{0}\right)$ can be partitioned into at most $N_{n}$ sets, each of which is $(n, \varepsilon)$-separated. It follows that $T^{-n}\left(x_{0}\right)$ contains a subset $\mathscr{N}$ that is $(n, \varepsilon)$-separated and such that

$$
\sum_{y \in \mathscr{N}} \exp \left(S_{n}(\varphi(y))\right) \geq \frac{1}{N_{n}} \mathscr{L}_{\varphi}^{n}(\mathbf{1})\left(x_{0}\right) .
$$

Thus, to prove inequality (4.2) it is enough to prove that $\lim \sup _{n \rightarrow+\infty} \frac{1}{n} \log N_{n}$ can be made arbitrarily small by taking $\varepsilon$ sufficiently small.

Let $L \geq 1$ be given. As none of the critical points of $T$ in $J(T)$ is periodic, there is $\varepsilon>0$ such that for every $c \in \operatorname{Crit}(T) \cap J(T), x \in B(c, 2 \varepsilon)$ and $j \in\{1, \ldots, L\}$ we have $T^{j}(x) \notin B(c, 2 \varepsilon)$. Reducing $\varepsilon$ if necessary we assume that for every $x \in J(T)$ such that $\operatorname{dist}(x, \operatorname{Crit}(T) \cap J(T)) \geq 2 \varepsilon$, the map $T$ is injective on $B(x, \varepsilon)$.

For each $y \in J(T)$ put $N_{0}(y)=1$. Note that if $y_{0} \in J(T)$ and $y \in$ $T^{-n}\left(T^{n}\left(y_{0}\right)\right)$ are $(n, \varepsilon)$-close, then $T(y)$ and $T\left(y_{0}\right)$ are $(n-1, \varepsilon)$-close. So we have $N_{n}\left(y_{0}\right) \leq \operatorname{deg}(T) N_{n}\left(T\left(y_{0}\right)\right)$, and when $T$ is injective on $B\left(y_{0}, \varepsilon\right)$ we have $N_{n}\left(y_{0}\right)=N_{n-1}\left(T\left(y_{0}\right)\right)$. In particular we have $N_{n}\left(y_{0}\right)=N_{n-1}\left(T\left(y_{0}\right)\right)$ when $\operatorname{dist}\left(y_{0}, \operatorname{Crit}(T) \cap J(T)\right) \geq 2 \varepsilon$. By induction and the definition of $L$ we obtain that

$$
N_{n}\left(y_{0}\right) \leq \operatorname{deg}(T)^{\#(\operatorname{Crit}(T) \cap J(T))(1+n / L)},
$$

and that

$$
\limsup _{n \rightarrow+\infty} \frac{1}{n} N_{n}\left(y_{0}\right) \leq L^{-1} \#(\operatorname{Crit}(T) \cap J(T)) \log \operatorname{deg}(T) .
$$


As we can take $L$ arbitrarily large by taking $\varepsilon>0$ sufficiently close to 0 , this completes the proof of the desired assertion.

3. We will complete the proof of the lemma. By part 1 of Lemma 4.1 there is $\lambda>1$ and a probability measure $\eta$ such that $\mathscr{L}_{\varphi}^{*}(\eta)=\lambda \eta$. Then for every integer $n \geq 1$ we have

$$
\int \mathscr{L}_{\varphi}^{n}(\mathbf{1}) d \eta=\int 1 d\left(\mathscr{L}_{\varphi}^{*}\right)^{n}(\eta)=\lambda^{n}
$$

Thus, by part 1 we have that for every $x \in J(T)$,

$$
C_{0}^{-1} \lambda^{n} \leq \mathscr{L}_{\varphi}^{n}(\mathbf{1})(x) \leq C_{0} \lambda^{n} .
$$

Part 2 implies then that $\lambda=\exp (P(T, \varphi))$.

Lemma 4.3. Let $T$ be a rational map satisfying the TCE condition, and for each integer $n \geq 1$ put $\operatorname{Per}_{n}=\left\{p \in J(T) \mid T^{n}(p)=p\right\}$. Then for every Hölder continuous function $\varphi: J(T) \rightarrow \mathbb{R}$ we have

$$
\lim _{n \rightarrow+\infty} \frac{1}{n} \log \sum_{p \in \operatorname{Per}_{n}} \exp \left(S_{n}(\varphi)(p)\right)=P(T, \varphi) .
$$

Proof. Let $\lambda_{\mathrm{ESC}}>1$ and $r_{0}>0$ be as in the ESC condition, and let $\kappa \in(0,1)$ be the exponent of $\varphi$. Just as in the proof of Lemma 4.2 we will use the following fact several times: Letting $C:=|\varphi|_{\kappa}\left(2 r_{0}\right)^{\kappa}\left(\lambda_{\mathrm{ESC}}^{\kappa}-1\right)^{-1}$, for each $x, x^{\prime} \in J(T)$ that belong to a ball $B$ of radius less than or equal to $r_{0}$ centered at $J(T)$, and for each $y \in T^{-n}(x)$ and $y^{\prime} \in T^{-n}\left(x^{\prime}\right)$ in the same connected component of $T^{-n}(B)$, we have $\left|S_{n}(\varphi)(y)-S_{n}(\varphi)\left(y^{\prime}\right)\right| \leq C$.

Let $n_{0} \geq 1$ be sufficiently large so that $\lambda_{\mathrm{ESC}}^{n_{0}}<r_{0} / 3$, and fix $n \geq n_{0}$.

Let $F$ be a finite subset of $J(T)$ that is $\left(r_{0} / 3\right)$-dense in $J(T)$. Let $x \in F$ and let $W$ be a connected component of $T^{-n}\left(B\left(x, r_{0}\right)\right)$ intersecting $B\left(x, r_{0} / 3\right)$. We have $W \subset B\left(x, 2 r_{0} / 3\right)$, so the number of elements of $\mathrm{Per}_{n}$ contained in $W$ is the same as the number of elements of $T^{-n}\left(x_{0}\right)$ in $W$, counted with multiplicity. Considering that each element of $\operatorname{Per}_{n}$ is contained in such a $W$, we conclude that

$$
\sum_{p \in \operatorname{Per}_{n}} \exp \left(S_{n}(\varphi)(p)\right) \leq \exp (C) \sum_{x \in F} \mathscr{L}_{\varphi}^{n}(\mathbf{1})(x) .
$$

Then Lemma 4.2 implies that

$$
\limsup _{n \rightarrow+\infty} \frac{1}{n} \log \sum_{p \in \operatorname{Per}_{n}} \exp \left(S_{n}(\varphi)(p)\right) \leq P(T, \varphi) .
$$

Fix $x_{0} \in J(T)$ and let $m_{0} \geq 1$ be sufficiently large so that $J(T) \subset$ $T^{m_{0}}\left(B\left(x_{0}, r_{0} / 3\right)\right)$. Let $W$ be a connected component of $T^{-n}\left(B\left(x_{0}, r_{0}\right)\right)$. Then there is a connected component $W_{0}$ of $T^{-m_{0}}(W)$ intersecting $B\left(x_{0}, r_{0} / 3\right)$. Since $W_{0}$ is a connected component of $T^{-\left(n+m_{0}\right)}\left(B\left(x_{0}, r_{0}\right)\right)$, we have $W_{0} \subset$ $B\left(x_{0}, 2 r_{0} / 3\right)$. So, if we denote by $D_{0}$ the degree of $T^{n+m_{0}}: W_{0} \rightarrow B\left(x_{0}, r_{0}\right)$, then $W_{0}$ contains precisely $D_{0}$ elements of $\operatorname{Per}_{n+m_{0}}$. Since the degree of $T^{n}$ : 
$W \rightarrow B\left(x_{0}, r_{0}\right)$ is less than or equal to $D_{0}$, letting $C^{\prime}=\exp \left(C+m_{0} \sup _{\overline{\mathbb{C}}}|\varphi|\right)$, we have

$$
\sum_{x \in W \cap T^{-n}\left(x_{0}\right)} \operatorname{deg}_{T}(x) \exp \left(S_{n}(\varphi)\right)(x) \leq C^{\prime} \sum_{p \in W_{0} \cap \operatorname{Per}_{n+m_{0}}} \exp \left(S_{n}(\varphi)(p)\right) .
$$

We thus have

$$
\sum_{p \in \operatorname{Per}_{n+m_{0}}} \exp \left(S_{n}(\varphi)(p)\right) \geq\left(C^{\prime}\right)^{-1} \mathscr{L}_{\varphi}^{n}(\mathbf{1})\left(x_{0}\right)
$$

and Lemma 4.2 implies that

$$
\liminf _{n \rightarrow+\infty} \frac{1}{n} \log \sum_{p \in \operatorname{Per}_{n}} \exp \left(S_{n}(\varphi)(p)\right) \geq P(T, \varphi) .
$$

Lemma 4.4. Let $T$ be a complex rational map satisfying the TCE condition, let $\varphi: J(T) \rightarrow \mathbb{R}$ be a Hölder continuous function, and let $\mu_{\varphi}$ be the unique equilibrium state of $T$ for the potential $\varphi$. Then for every Hölder continuous function $\psi: J(T) \rightarrow \mathbb{R}$ we have

$$
\lim _{n \rightarrow+\infty} \frac{1}{n} \log \int \exp \left(S_{n}(\psi)\right) d \mu_{\varphi}=P(T, \varphi+\psi)-P(T, \varphi) .
$$

Proof. Let $\eta_{\varphi}$ be the $(\varphi-P(T, \varphi))$-conformal measure of $T$, and let $h_{\varphi}$ be the Radon-Nikodym derivative of $\mu_{\varphi}$ with respect to $\eta_{\varphi}$. Since inf $h_{\varphi}>0$ and $\sup h_{\varphi}<+\infty$, it is enough to prove the limit with $\mu_{\varphi}$ replaced by $\eta_{\varphi}$.

For each integer $n \geq 1$ we have

$$
\begin{aligned}
& \int \exp \left(S_{n}(\psi)\right) d \eta_{\varphi}=\int \exp (\left.S_{n}(\psi)\right) d\left(\exp (-n P(T, \varphi)) \mathscr{L}_{\varphi}^{* n} \eta_{\varphi}\right) \\
&=\exp (-n P(T, \varphi)) \int \mathscr{L}_{\varphi}^{n}\left(\exp \left(S_{n}(\psi)\right)\right) d \eta_{\varphi}
\end{aligned}
$$

Using $\mathscr{L}_{\varphi}^{n}\left(\exp \left(S_{n}(\psi)\right)\right)=\mathscr{L}_{\varphi+\psi}^{n} \mathbf{1}$, the assertion of the proposition is then a direct consequence of Lemma 4.2 .

\subsection{Proof of Theorem $\mathrm{B}$ and its corollaries.}

Proof of Theorem $B$. First recall that the topological entropy of $T$ is equal to $\log \operatorname{deg}(T)$ Gro03, Lju83, and that the measure-theoretic entropy of $T$ is upper semi-continuous FLM83, Lju83. Fix a Hölder continuous function $\psi: J(T) \rightarrow \mathbb{R}$. For the sequence $\left(\Omega_{n}\right)_{n \geq 1}$ associated to periodic points we 
have,

$$
\begin{aligned}
\int_{\mathscr{M}(J(T))} \exp \left(n \int \psi d \mu\right) d \Omega_{n}(\mu) & \\
= & \frac{\sum_{p \in \operatorname{Per}_{n}} \exp \left(S_{n}(\varphi)(p)\right) \exp \left(n \int \psi d W_{n}(p)\right)}{\sum_{p^{\prime} \in \operatorname{Per}_{n}} \exp \left(S_{n}(\varphi)\left(p^{\prime}\right)\right)} \\
= & \frac{\sum_{p \in \operatorname{Per}_{n}} \exp \left(S_{n}(\varphi+\psi)(p)\right)}{\sum_{p^{\prime} \in \mathrm{Per}_{n}} \exp \left(S_{n}(\varphi)\left(p^{\prime}\right)\right)} .
\end{aligned}
$$

Analogously, for the sequence $\left(\Omega_{n}\left(x_{0}\right)\right)_{n \geq 1}$ associated to the iterated preimages of a point $x_{0} \in J(T)$, we have

$$
\int_{\mathscr{M}(J(T))} \exp \left(n \int \psi d \mu\right) d \Omega_{n}\left(x_{0}\right)(\mu)=\frac{\sum_{x \in T^{-n}\left(x_{0}\right)} \exp \left(S_{n}(\varphi+\psi)(x)\right)}{\sum_{y \in T^{-n}\left(x_{0}\right)} \exp \left(S_{n}(\varphi)(y)\right)} .
$$

Finally, for the sequence $\left(\Sigma_{n}\right)_{n \geq 1}$ associated to the Birkhoff averages we have,

$$
\int_{\mathscr{M}(J(T))} \exp \left(n \int \psi d \mu\right) d \Sigma_{n}(\mu)=\int_{J(T)} \exp \left(S_{n}(\psi)\right) d \mu_{\varphi}
$$

Therefore, (1.5) holds with $\psi$ for the sequences $\left(\Omega_{n}\right)_{n \geq 1},\left(\Omega_{n}\left(x_{0}\right)\right)_{n \geq 1}$, and $\left(\Sigma_{n}\right)_{n \geq 1}$, by Lemma 4.3, Lemma 4.2, and Lemma 4.4, respectively. Consequently, all the assertions of Theorem $\mathrm{B}$ follow from Theorem $\mathrm{A}$ and Theorem $\mathrm{C}$.

Proof of Corollary 1.1. The first assertion is obtained from Theorem B applying the contraction principle with the map $\widehat{\psi}$ (see $\S 2$. For each $\delta>0$ put

$$
\mathscr{G}_{1, \delta}=\left\{\mu \in \mathscr{M}(J(T)): \int \psi d \mu>\delta\right\}
$$

and

$$
\mathscr{G}_{2, \delta}=\left\{\mu \in \mathscr{M}(J(T)): \int \psi d \mu<-\delta\right\} .
$$

If there exists some $\delta_{0}>0$ such that $\left(\mathscr{G}_{1, \delta_{0}} \cup \mathscr{G}_{2, \delta_{0}}\right) \cap \mathscr{M}(J(T), T) \neq \emptyset$, then (1.4) follows from the last statement of Theorem B for all $\varepsilon \in\left(0, \delta_{0}\right]$. Moreover, the value of (1.4) is strictly negative since by hypothesis $\mu_{\varphi} \notin$ $\overline{\mathscr{G}_{1, \varepsilon} \cup \mathscr{G}_{2, \varepsilon}}$. Assume now that for all $\delta>0$ we have $\left(\mathscr{G}_{1, \delta} \cup \mathscr{G}_{2, \delta}\right) \cap \mathscr{M}(J(T), T)=$ $\emptyset$. Since for each $\delta>0$ and $j \in\{1,2\}$ we have $\overline{\mathscr{G}_{j, 2 \delta}} \subset \mathscr{G}_{j, \delta}$, we obtain

$$
\overline{\mathscr{G}_{1, \delta} \cup \mathscr{G}_{2, \delta}} \cap \mathscr{M}(J(T), T)=\emptyset,
$$

and the conclusion follows from the large deviation upper bounds applied to $\overline{\mathscr{G}_{1, \delta} \cup \mathscr{G}_{2, \delta}}$ for all $\delta>0$ (so that both sides of (1.4) are $-\infty$ ).

Proof of Corollary 1.2. The first (resp. second) equality is a direct consequence of the definition of the rate function (1.2) together with (1.3), 
and Lemma 4.3 (resp. Lemma 4.2). The last equality follows from (1.2) and (1.3).

\section{Appendix A. A Ruelle-Perron-Frobenius type theorem for TCE RATIONAL MAPS}

The purpose of this appendix is to give an alternative proof of Theorem $\mathrm{A}$, as a direct consequence of the following Ruelle-Perron-Frobenius type theorem; compare with [DU91a, Prz90, DPU96.

Theorem D. Let $T$ be a rational map satisfying the TCE condition and let $\varphi: J(T) \rightarrow \mathbb{R}$ be a Hölder continuous function. Then the following conclusions hold.

1. There is a unique probability measure $\eta_{0}$ that is supported on $J(T)$ and that satisfies

$$
\mathscr{L}_{\varphi}^{*} \eta_{0}=\exp (P(T, \varphi)) \eta_{0}
$$

More generally, if for some $\lambda>1$ there is a probability measure $\eta$ supported on $J(T)$ and such that $\mathscr{L}_{\varphi}^{*} \eta=\lambda \eta$, then $\lambda=\exp (P(T, \varphi))$ and $\eta=\eta_{0}$.

In particular $\eta_{0}$ is the unique $(\exp (P(T, \varphi)-\varphi))$-conformal probability measure for $T$ supported on $J(T)$.

2. There is a unique Hölder continuous function $h_{0}: J(T) \rightarrow(0,+\infty)$ satisfying

$$
\mathscr{L}_{\varphi} h_{0}=\exp (P(T, \varphi)) h_{0} \text { and } \int h_{0} d \eta_{0}=1 .
$$

Furthermore, the probability measure $h_{0} \eta_{0}$ is invariant by $T$ and it is the unique equilibrium state of $T$ for the potential $\varphi$.

To prove this result we first consider the following lemma, which is precisely [PRL07, Part 1 of Lemma 3.3].

Lemma A.1. Let $T$ be a rational map satisfying the ESC condition with constants $\lambda_{\mathrm{ESC}}>1$ and $r_{0}>0$. Then there are constants $\theta_{0} \in(0,1)$ and $C_{0}>0$ such that for each $x \in J(T)$, each $r \in\left(0, r_{0}\right)$, and each connected component $W$ of $T^{-n}(B(x, r))$, we have

$$
\operatorname{diam}(W) \leq C_{0} \lambda_{\mathrm{ESC}}^{-n} r^{\theta_{0}} .
$$

We denote by $\|\cdot\|_{\infty}$ the supremum norm on the space of real continuous functions defined on $J(T)$. Given $\alpha \in(0,1]$ we will say that a function $\varphi$ : $J(T) \rightarrow \mathbb{R}$ is Hölder continuous with exponent $\alpha$ if there is a constant $C>0$ such that for all $x, y \in J(T)$ we have

$$
|\varphi(x)-\varphi(y)| \leq C \operatorname{dist}(x, y)^{\alpha} .
$$

For such a function $\varphi$ we put

$$
|\varphi|_{\alpha}=\sup \left\{|\varphi(x)-\varphi(y)| \operatorname{dist}(x, y)^{-\alpha}: x, y \in J(T) \text { distinct }\right\} \text {, }
$$


and $\|\varphi\|_{\alpha}=\|\varphi\|_{\infty}+|\varphi|_{\alpha}$. Note that $\|\varphi\|_{\alpha}$ defines a norm on the space of Hölder continuous functions with exponent $\alpha$.

Lemma A.2. Let $T$ be a rational map satisfying the ESC condition, and let $\theta_{0} \in(0,1)$ be given by Lemma A.1. Then for every $\alpha \in(0,1)$, and every Hölder continuous function $\varphi: J(T) \rightarrow \mathbb{R}$ with exponent $\alpha$, there is a constant $C>0$ such that for every $\beta \in(0, \alpha]$, every Hölder continuous function $\psi: J(T) \rightarrow \mathbb{R}$ with exponent $\beta$, and every integer $n \geq 1$ we have

$$
\left\|\mathscr{L}_{\varphi}^{n} \psi\right\|_{\beta \theta_{0}} \leq C \exp (n P(T, \varphi))\left(\|\psi\|_{\infty}+\lambda_{\mathrm{ESC}}^{-n \beta}|\psi|_{\beta}\right) .
$$

Proof. Let $\lambda_{\mathrm{ESC}}$ and $r_{0}>0$ be as in the ESC condition.

Let $x, x^{\prime} \in J(T)$ be outside of the forward orbits of the critical points of $T$, and fix an integer $n$. Observe that each connected component of $T^{-n}\left(B\left(x, r_{0}\right)\right)$ contains the same number of elements of $T^{-n}(x)$ and of $T^{-n}\left(x^{\prime}\right)$. Therefore there is a bijection $\iota: T^{-n}(x) \rightarrow T^{-n}\left(x^{\prime}\right)$ such that for every $y \in T^{-n}(x)$, both $y$ and $\iota(y)$ belong to the same connected component of $T^{-n}\left(B\left(x, r_{0}\right)\right)$. In particular we have

$$
\operatorname{dist}(y, \iota(y)) \leq C_{0} \lambda_{\mathrm{ESC}}^{-n} \operatorname{dist}\left(x, x^{\prime}\right)^{\theta_{0}} .
$$

Using Lemma A.1, we obtain that for each $y \in T^{-n}(x)$ we have

$$
\left|S_{n}(\varphi)(y)-S_{n}(\varphi)(\iota(y))\right| \leq|\varphi|_{\alpha} C_{0}^{\alpha}\left(\lambda_{\mathrm{ESC}}^{\alpha}-1\right)^{-1} \operatorname{dist}\left(x, x^{\prime}\right)^{\theta_{0} \alpha} .
$$

So, if we put $C_{1}=|\varphi|_{\alpha} C_{0}^{\alpha}\left(\lambda_{\mathrm{ESC}}^{\alpha}-1\right)^{-1}$, then we have

$\left|\exp \left(S_{n}(\varphi)(y)\right)-\exp \left(S_{n}(\varphi)\left(y^{\prime}\right)\right)\right| \leq \exp \left(C_{1} r_{0}^{\theta_{0} \alpha}\right) C_{1} \exp \left(S_{n}(\varphi)(y)\right) \operatorname{dist}\left(x, x^{\prime}\right)^{\theta_{0} \alpha}$.

Using this inequality we obtain,

$$
\begin{aligned}
& \left|\mathscr{L}_{\varphi}^{n} \psi(x)-\mathscr{L}_{\varphi}^{n} \psi\left(x^{\prime}\right)\right| \leq \\
& \leq \mid \sum_{y \in T^{-n}(x)}\left(\exp \left(S_{n}(\varphi)(y)\right)-\exp \left(S_{n}(\varphi)(\iota(y))\right) \psi(y) \mid+\right. \\
& +\left|\sum_{y \in T^{-n}(x)} \exp \left(S_{n}(\varphi)(y)\right)(\psi(y)-\psi(\iota(y)))\right| \leq \\
& \leq \mathscr{L}_{\varphi}^{n}|\psi|(x) \exp \left(C_{1} r_{0}^{\theta_{0} \alpha}\right) C_{1} \operatorname{dist}\left(x, x^{\prime}\right)^{\theta_{0} \alpha}+ \\
& +\mathscr{L}_{\varphi} \mathbf{1}(x)|\psi|{ }_{\beta} C_{0}^{\beta} \lambda_{\mathrm{ESC}}^{-n \beta} \operatorname{dist}\left(x, x^{\prime}\right)^{\theta_{0} \beta} .
\end{aligned}
$$

Since the union of the forward orbits of critical points of $T$ is nowhere dense in $J(T)$, we conclude that the last inequality holds for every $x, x^{\prime} \in$ $J(T)$. Then the assertion of the lemma is obtained using Lemma 4.2.

Proof of Theorem $D$. Let $\alpha \in(0,1)$ be the exponent of $\varphi$, and let $\theta_{0} \in(0,1)$ be given by Lemma A.1. 
1. Let $\psi: J(T) \rightarrow \mathbb{R}$ be a given Hölder continuous function with exponent $\alpha$. For each integer $n \geq 1$ put

$$
\psi_{n}:=\frac{1}{n} \sum_{k=0}^{n-1} \exp (-k P(T, \varphi)) \mathscr{L}_{\varphi}^{k} \psi .
$$

Then Lemma A.2 implies that the sequence $\left(\left\|\psi_{n}\right\|_{\alpha \theta_{0}}\right)_{n \geq 1}$ is bounded from above independently of $n$. It follows that there is a sequence of positive integers $\left(n_{j}\right)_{j \geq 1}$, such that $\left(\psi_{n_{j}}\right)_{j \geq 1}$ converges uniformly to a Hölder continuous function $\psi_{0}$ of exponent $\alpha \theta_{0}$. We thus have

$$
\mathscr{L}_{\varphi} \psi_{0}=\exp (P(T, \varphi)) \psi_{0}
$$

2. Denote by $h_{0}$ a function $\psi_{0}$ as in part 1 when $\psi=\mathbf{1}$. Lemma 4.2 implies that $h_{0}$ takes values in $\left[C_{0}^{-1}, C_{0}\right] \subset(0,+\infty)$.

We will show that for every $\psi$ and $\psi_{0}$ as in part 1 the function $\psi_{0} / h_{0}$ is constant. Put

$$
C:=\sup \left\{\psi_{0}(x) h_{0}(x)^{-1}: x \in J(T)\right\},
$$

and let $X$ be the compact set of those $x \in J(T)$ such that $\psi_{0}(x)=C h_{0}(x)$. Then for $x \in X$ we have

$$
\begin{aligned}
& C \exp (P(T, \varphi)) h_{0}(x)=\exp (P(T, \varphi)) \psi_{0}(x)= \\
& =\sum_{y \in T^{-1}(x)} \operatorname{deg}_{T}(y) \exp (\varphi(y)) \psi_{0}(y) \leq C \sum_{y \in T^{-1}(x)} \operatorname{deg}_{T}(y) \exp (\varphi(y)) h_{0}(y)= \\
& =C \exp (P(T, \varphi)) h_{0}(x),
\end{aligned}
$$

which implies that $T^{-1}(X) \subset X$. Therefore $T^{-1}(X) \subset X$, and by the locally eventually onto property of $T$ on $J(T)$ we have that $X=J(T)$. That is, we have $\psi_{0}=C h_{0}$, as wanted.

3. Let $\lambda>0$ and let $\eta_{0}$ be a probability measure supported on $J(T)$ such that $\mathscr{L}_{\varphi}^{*} \eta_{0}=\lambda \eta_{0}$. Part 1 of Lemma 4.1 guaranties that there is at least one such $\lambda$ and $\eta_{0}$. Note that for every integer $n \geq 1$ we have

$$
\int \mathscr{L}_{\varphi}^{n} 1 d \eta_{0}=\int 1 d \mathscr{L}_{\varphi}^{*} \eta_{0}=\lambda^{n}
$$

so Lemma 4.2 implies that $\lambda=\exp (P(T, \varphi))$ and hence that $\mathscr{L}_{\varphi}^{*} \eta_{0}=$ $\exp (P(T, \varphi)) \eta_{0}$.

Note that for each $\psi$ and $\psi_{0}$ as in part 1 we have $\int \psi_{0} d \eta_{0}=\int \psi d \eta_{0}$. In particular, letting $\psi=1$, we obtain that $\int h_{0} d \eta_{0}=1$. If we denote by $C>0$ the constant given by part 2 , so that $\psi_{0}=C h_{0}$, then we have

$$
\int \psi d \eta_{0}=\int \psi_{0} d \eta_{0}=\int C h_{0} d \eta_{0}=C .
$$

That is, we have shown that for each accumulation point $\psi_{0}$ of the sequence of functions $\left(\psi_{n}\right)_{n \geq 1}$ defined in part 1 , we have $\psi_{0}=\left(\int \psi d \eta_{0}\right) h_{0}$. As this 
property determines $\eta_{0}$ uniquely, we conclude that $\eta_{0}$ is the unique probability measure $\eta$ that is supported on $J(T)$ and for which there is $\lambda>0$ such that $\mathscr{L}_{\varphi}^{*} \eta=\lambda \eta$.

4. To show that the measure $h_{0} \eta_{0}$ is invariant by $T$, observe that for each continuous function $\psi: J(T) \rightarrow \mathbb{R}$ we have

$$
\begin{aligned}
& \int \psi d T\left[h_{0} \eta_{0}\right]= \int \psi \circ T d h_{0} \eta_{0} \\
&= \int \psi \circ T \cdot h_{0} d\left(\exp (-P(T, \varphi)) \mathscr{L}_{\varphi}^{*} \eta_{0}\right) \\
&= \exp (-P(T, \varphi)) \int \mathscr{L}_{\varphi}\left(\psi \circ T \cdot h_{0}\right) d \eta_{0} \\
& \quad=\exp (-P(T, \varphi)) \int \psi \mathscr{L}_{\varphi} h_{0} d \eta_{0}=\int \psi h_{0} d \eta_{0} .
\end{aligned}
$$

Let $\rho$ be an ergodic and invariant probability measure supported on $J(T)$. We will show that $\rho$ is an equilibrium state of $T$ for the potential $\varphi$ if and only if the measure $\eta:=h_{0}^{-1} \rho$ is $(\exp (P(T, \varphi)-\varphi))$-conformal for $T$. Together with the uniqueness of $\eta_{0}$, this implies that the measure $h_{0} \eta_{0}$ is the unique equilibrium state of $T$ for the potential $\varphi$.

As $T$ satisfies the TCE condition, the Lyapunov exponent of $\rho$ is positive [PRLS03, Main Theorem], so $\rho$ admits a generating partition of finite entropy, see for example Mañ83] (where it was assumed that the entropy is positive, but in fact it was only used that the Lyapunov exponent is positive), DU91a, §2], Dob08] or [PU02. This implies that Rokhlin formula holds [Par69, 10§]:

$$
h_{\rho}=\int \log \operatorname{Jac}_{\rho} d \rho .
$$

Using $\mathrm{Jac}_{\eta}=\frac{h_{0} \circ T}{h_{0}} \mathrm{Jac}_{\rho}$, we obtain $h_{\rho}=\int \log \mathrm{Jac}_{\eta} d \rho$, and

$$
\begin{aligned}
h_{\rho} & -P(T, \varphi)+\int \varphi d \rho \\
& =\int \log \left(\operatorname{Jac}_{\eta} \exp (\varphi-P(T, \varphi))\right) d \rho \\
& \leq \int \operatorname{Jac}_{\eta} \exp (\varphi-P(T, \varphi)) d \rho-1 \\
& =\exp (-P(T, \varphi)) \int \sum_{y \in T^{-1}(x)} \operatorname{Jac}_{\rho}(y)^{-1} \operatorname{Jac}_{\eta}(y) \exp (\varphi(y)) d \rho(x)-1 \\
& =\exp (-P(T, \varphi)) \int h_{0}(x)^{-1} \sum_{y \in T^{-1}(x)} h_{0}(y) \exp (\varphi(y)) d \rho(x)-1 \\
& =\exp (-P(T, \varphi)) \int h_{0}^{-1} \mathscr{L}_{\varphi} h_{0} d \rho-1 \\
& =0
\end{aligned}
$$


This shows that $\rho$ is an equilibrium state of $T$ for the potential $\varphi$ if and only if $\operatorname{Jac}_{\eta}=\exp (P(T, \varphi)-\varphi)$ holds on a set of full measure with respect to $\rho$. Since $h_{0}$ takes values on $\left[C_{0}^{-1}, C_{0}\right]$, this last condition is equivalent to the condition that $\operatorname{Jac}_{\eta}=\exp (P(T, \varphi))$ holds on a set of full measure with respect to $\eta$; or equivalently, that $\eta$ is a $\exp (P(T, \varphi)-\varphi)$-conformal measure for $T$.

\section{Appendix B. On KifER's Result FOR SEMi-FlOWs}

In this section we clarify the relation between Theorem $[$ and the main result of [Kif90] concerning dynamical systems (namely, Theorem 3.4 of that paper). We claim no originality concerning the proofs of Theorem $\mathrm{C}$ and Theorem E, since in both cases the basic ideas are in [Kif90]. See [Ara07] and references therein for large deviation upper-bounds, for some non-uniformly hyperbolic semi-flows.

Recall that Kif90 concerns large deviations in $\mathscr{M}(Y)$, where $Y$ is a compact metric space that is not necessarily invariant. We show how the large deviation lower bounds in $\mathscr{M}(Y)$ can be recovered, and in fact slightly strengthened (see Remark B.1), from Theorem Cand Remark B.2. In order to get the upper bounds in $\mathscr{M}(Y)$ we use the extension of the variational principe proved in [Kif90]. However, if we consider the closure $X$ of the union of the supports of all the invariant probability measures on $Y$, then $X$ is invariant and the large deviation principle will be obtained in $\mathscr{M}(X)$ from Theorem C.

The basic ingredients are the following. Let $M$ be a locally compact metric space, let $Y$ be a compact subset of $M$, and let $\mathfrak{T} \in\left\{\mathbb{Z}_{+}, \mathbb{R}_{+}\right\}$. For each $t \in \mathfrak{T}$ let $F^{t}: M \rightarrow M$ be a continuous map, put $Y_{t}=\left\{x \in M: F^{s}(x) \in\right.$ $Y, 0 \leq s \leq t\}, \mathscr{M}_{Y}^{F}=\left\{\mu \in \mathscr{M}(Y): F^{t}[\mu]=\mu, t \geq 0\right\}$ (i.e. $\mathscr{M}_{Y}^{F}$ is the set of $F^{t}$-invariant probability measures for all $\left.t \in \mathfrak{T}\right)$, and $X=\overline{\bigcup_{\mu \in \mathscr{M}_{Y}^{F}} \operatorname{supp} \mu}$. We shall use the notations of Remark B.2 for the system induced on $X$; more precisely, let $\tau$ be the action of $\mathfrak{T}$ on $X$ given by $\tau^{t}=F^{t}$ for all $t \in \mathfrak{T}$, so that $X$ is $\tau$-invariant with $\mathscr{M}^{\tau}(X)=\mathscr{M}_{Y}^{F}$. When $\mathscr{M}_{Y}^{F} \neq \emptyset$, for each $\mu \in \mathscr{M}_{Y}^{F}$ let $h_{\mu}^{1}$ denote the entropy of $F^{1}$ with respect to $\mu$, and note that $h_{\mu}^{\tau}=h_{\mu}^{1}$. For each $\phi \in C(Y)$ let $\tilde{I}^{\phi}$ be the function defined on $\mathscr{M}(Y)$ by

$$
\tilde{I}^{\phi}(\mu)= \begin{cases}P^{\tau}\left(\phi_{\mid X}\right)-\int \phi d \mu-h_{\mu}^{1} & \text { if } \mu \in \mathscr{M}_{Y}^{F} ; \\ +\infty & \text { if } \mu \in \mathscr{M}(Y) \backslash \mathscr{M}_{Y}^{F} .\end{cases}
$$

Since $\mathscr{M}^{\tau}(X)=\mathscr{M}_{Y}^{F}$ and $h_{\mu}^{\tau}=h_{\mu}^{1}$, by identifying $\mathscr{M}(X)$ as a (closed) subset of $\mathscr{M}(Y)$ we see that $\tilde{I}^{\phi}$ coincides on $\mathscr{M}(X)$ (and takes infinite value outside) with the function $I^{\phi_{\mid X}}$ associated to the system $(X, \tau)$ as in Remark B.2. defined by

$$
I^{\phi_{\mid X}}(\mu)= \begin{cases}P^{\tau}\left(\phi_{\mid X}\right)-\int \phi d \mu-h_{\mu}^{\tau} & \text { if } \mu \in \mathscr{M}^{\tau}(X) \\ +\infty & \text { if } \mu \in \mathscr{M}(X) \backslash \mathscr{M}^{\tau}(X) .\end{cases}
$$


For each $t \in \mathfrak{T}$ let $W_{t}: Y_{t} \mapsto \mathscr{M}\left(Y_{t}\right)$ defined by $W_{t}(x)=\frac{1}{t} \int_{0}^{t} \delta_{F^{t}(x)} d s$ when $\mathfrak{T}=\mathbb{R}_{+}$, and $W_{t}(x)=\frac{1}{t} \sum_{i=0}^{t-1} \delta_{F^{t}(x)}$ when $\mathfrak{T}=\mathbb{Z}_{+}$.

Theorem E (Following Kifer). Let $m \in \mathscr{M}(Y)$, let $\phi \in C(Y)$, and assume that the following conditions hold.

(i) $\mathscr{M}_{Y}^{F} \neq \emptyset$ and the map $h^{1}$ on $\mathscr{M}_{Y}^{F}$ is finite and upper semi-continuous;

(ii) For each $t \in \mathfrak{T}$, each $x \in X$ and each $\delta>0$ we have

$$
a_{\delta, t}{ }^{-1} \leq m\left(U_{\delta, x, t}\right) \exp \left(-t \int_{Y} \phi d W_{t}(x)\right) \leq a_{\delta, t}
$$

where

$$
U_{\delta, x, t}=\left\{y \in Y_{t}: d\left(F^{u}(x), F^{u}(y)\right) \leq \delta, 0 \leq u \leq t\right\}
$$

and $a_{\delta, t}$ satisfies

$$
\lim _{\delta \rightarrow 0} \lim _{t \rightarrow+\infty} a_{\delta, t}^{1 / t}>0
$$

The following conclusions hold.

1. For each closed subset $\mathscr{F}$ of $\mathscr{M}(X)$ we have

$$
\limsup _{t \rightarrow+\infty} \frac{1}{t} \log m\left\{x \in X: W_{t}(x) \in \mathscr{F}\right\} \leq-\inf _{\mathscr{F}} \tilde{I}^{\phi} .
$$

If moreover

$$
m\left(U_{\delta, x, t}\right) \exp \left(-t \int_{Y} \phi d W_{t}(x)\right) \leq a_{\delta, t}
$$

for all $t \in \mathfrak{T}$, all $x \in Y_{t}$ and all $\delta>0$, then we can replace $X$ by $Y$ in the above assertion.

2. If there is a dense vector subspace $\mathcal{W} \subset C(X)$ such that for each $\psi \in \mathcal{W}$ there is a unique measure $\mu \in \mathscr{M}(Y)$ realizing the supremum in $\sup _{\mu \in \mathscr{M}_{Y}^{F}}\left\{\int(\psi+\phi) d \mu+h_{\mu}^{1}\right\}$, then for each open subset $\mathscr{G}$ of $\mathscr{M}(Y)$ we have

$$
\begin{aligned}
\left.\liminf _{t \rightarrow+\infty} \frac{1}{t} \log m\left\{x \in Y: W_{t}(x) \in \mathscr{G}\right\}\right) & \\
\geq \liminf _{t \rightarrow+\infty} \frac{1}{t} \log m\left\{x \in X: W_{t}(x)\right. & \in \mathscr{G} \cap \mathscr{M}(X)\}) \\
& \geq-\inf _{\mathscr{G} \cap \mathscr{M}(X)} \tilde{I}^{\phi}=-\inf _{\mathscr{G}} \tilde{I}^{\phi} .
\end{aligned}
$$

Proof. Putting for each $\psi \in C(Y)$ and each $\delta>0$,

$$
\gamma_{\delta}(\psi)=\sup \{|\psi(y)-\psi(z)|: y \in Y, z \in Y, d(y, z) \leq \delta\},
$$

we get for each maximal $(\delta, t)$-separated set $S_{\delta, t}$ in $Y_{t}$,

$$
\frac{1}{t} \log \sum_{x \in S_{\delta, t} \cap X} m\left(U_{\delta / 2, x, t}\right) \exp \left(t \int_{X}\left(\psi-\gamma_{\delta}(\psi)\right) d W_{t}(x)\right)
$$




$$
\begin{gathered}
\leq \frac{1}{t} \log \int_{X} \exp \left(t \int_{X} \psi d W_{t}(x)\right) d m(x) \\
\left.\leq \frac{1}{t} \log \sum_{x \in S_{\delta, t} \cap X} m\left(U_{\delta / 2, x, t}\right) \exp \left(t \int_{X} \psi+\gamma_{\delta}(\psi)\right) d W_{t}(x)\right),
\end{gathered}
$$

and using (ii) yields

$$
\lim _{t \rightarrow+\infty} \frac{1}{t} \log \int_{X} \exp \left(t \int_{X} \psi d W_{t}(x)\right) d m(x)=P^{\tau}\left(\phi_{\mid X}+\psi_{\mid X}\right)+\lim _{\delta \rightarrow 0} \lim _{t \rightarrow+\infty} \frac{1}{t} \log a_{\delta, t}
$$

(note that $S_{\delta, t} \cap X$ is a maximal $(\delta, t)$-separated set in $X$ ). Taking $\psi=0$ in (B.2) gives

$$
\lim _{t \rightarrow+\infty} \frac{1}{t} \log m(X)=P^{\tau}\left(\phi_{\mid X}\right)+\lim _{\delta \rightarrow 0} \lim _{t \rightarrow+\infty} \frac{1}{t} \log a_{\delta, t}>-\infty
$$

which implies $m(X)>0$; in particular, both sides of the above equality vanish hence

$$
P^{\tau}\left(\phi_{\mid X}\right)=-\lim _{\delta \rightarrow 0} \lim _{t \rightarrow+\infty} \frac{1}{t} \log a_{\delta, t}
$$

We put $m_{X}=m / m(X)$, and shall consider the system $(X, \tau)$ and the net of image measures $\left(W_{t \mid X}\left[m_{X}\right]\right)$ on $\mathscr{M}(X)$. First note that the hypothesis (i) gives the upper semi-continuity of the map $h^{\tau}$. From $(\overline{B .2})$ and $(\overline{B .3})$ we obtain for each $\psi \in C(Y)$,

$\lim _{t \rightarrow+\infty} \frac{1}{t} \log \int_{\mathscr{M}(X)} \exp \left(t \int_{X} \psi d \mu\right) d W_{t \mid X}\left[m_{X}\right]=P^{\tau}\left(\phi_{\mid X}+\psi_{\mid X}\right)-P^{\tau}\left(\phi_{\mid X}\right)$.

Since any element of $C(X)$ is the restriction of some function in $C(Y)$, it follows that the general hypotheses of Com09, Theorem 5.2] hold for the net $\left(W_{t \mid X}\left[m_{X}\right]\right)$. Therefore, we get for each closed subset $\mathscr{F}$ of $\mathscr{M}(X)$,

$$
\begin{gathered}
\limsup _{t \rightarrow+\infty} \frac{1}{t} \log W_{t \mid X}\left[m_{X}\right](\mathscr{F})=\limsup _{t \rightarrow+\infty} \frac{1}{t} \log m\left\{x \in X: W_{t}(x) \in \mathscr{F}\right\} \\
\leq-\inf _{\mathscr{F}} I^{\phi_{\mid X}}=-\inf _{\mathscr{F}} \tilde{I}^{\phi}
\end{gathered}
$$

which proves the first assertion of part 1. Assume moreover that

$$
m\left(U_{\delta, x, t}\right) \exp \left(-t \int_{Y} \phi d W_{t}(x)\right) \leq a_{\delta, t}
$$

for all $t \in \mathfrak{T}$, all $x \in Y_{t}$ and all $\delta>0$. For each $t \in \mathfrak{T}$, let $m_{t}$ be the measure defined on $Y_{t}$ by putting $m_{t}=m / m\left(Y_{t}\right)$, and let $\bar{L}_{Y}$ be the large deviation functional associated to the net $\left(W_{t}\left[m_{t}\right]\right.$ ) (seen as acting on $\widetilde{\mathscr{M}}(Y)$ ). Replacing $S_{\delta, t} \cap X$ (resp. $X$ ) by $S_{\delta, t}$ (resp. $Y$ ) in the sums appearing in (B.1), and using (B.4) together with the fact that the topological pressure of any $\psi \in C(Y)$ coincides with $P^{\tau}\left(\psi_{\mid X}\right)$ ([Kif90, Proposition 3.1]) we get

$$
\bar{L}_{Y}(\widehat{\psi})=\limsup _{t \rightarrow+\infty} \frac{1}{t} \log \int_{Y_{t}} \exp \left(t \int_{Y} \psi d W_{t}(x)\right) d m(x)
$$




$$
\leq P^{\tau}\left(\phi_{\mid X}+\psi_{\mid X}\right)-P^{\tau}\left(\phi_{\mid X}\right)=Q_{\phi_{\mid X}}\left(\psi_{\mid X}\right),
$$

where $Q_{\phi_{\mid X}}$ is the map defined as in Lemma 3.1; moreover, by (B.2) and (B.3) the upper limit is a limit and the inequality is an equality, hence for each $\psi \in C(Y)$,

$$
L_{Y}(\widehat{\psi})=Q_{\phi_{\mid X}}\left(\psi_{\mid X}\right) .
$$

By [DZ98, Lemma 4.5.3], $\left(W_{t}\left[m_{t}\right]\right)$ satisfies the large deviation upper bounds in $\widetilde{\mathscr{M}}(Y)$ with the function

$$
\begin{aligned}
L_{Y}^{*}(\mu)= & \sup \left\{\mu(\psi)-L_{Y}(\widehat{\psi}): \psi \in C(Y)\right\}=\sup \left\{\mu(\psi)-Q_{\phi_{\mid X}}\left(\psi_{\mid X}\right): \psi \in C(Y)\right\} \\
& \geq \sup \left\{\mu_{\mid X}\left(\psi^{\prime}\right)-Q_{\phi_{\mid X}}\left(\psi^{\prime}\right): \psi^{\prime} \in C(X)\right\}=Q_{\phi_{\mid X}}^{*}\left(\mu_{\mid X}\right) .
\end{aligned}
$$

Since $\mathscr{M}(Y)$ is closed in $\widetilde{\mathscr{M}}(Y)$, the large deviation principle holds in $\mathscr{M}(Y)$ with rate function $L_{Y}{ }^{*} \mid \mathscr{M}(Y)$. Since the inequality in (B.5) is an equality when $\mu \in \mathscr{M}(X)$, we obtain $L_{Y}{ }^{*}{ }_{\mid M(Y)}=\tilde{I}^{\phi}$ by Lemma 3.1; this proves the last assertion of part 1 . The hypothesis in part 2 is equivalent to the one of Theorem [C (strictly speaking, of its analogue given by Remark B.2) by taking $\varphi=\phi_{\mid X}$. Consequently, we have for each open subset $\mathscr{G}^{\prime}$ of $\mathscr{M}(X)$,

$$
\begin{gathered}
\liminf _{t \rightarrow+\infty} \frac{1}{t} \log W_{t \mid X}\left[m_{X}\right]\left(\mathscr{G}^{\prime}\right)=\liminf _{t \rightarrow+\infty} \frac{1}{t} \log m\left\{x \in X: W_{t}(x) \in \mathscr{G}^{\prime}\right\} \\
\geq-\inf _{\mathscr{G}^{\prime}} I^{\phi_{\mid X}}=-\inf _{\mathscr{G}^{\prime}} \tilde{I}^{\phi},
\end{gathered}
$$

which proves the assertion of part 2 concerning $\mathscr{M}(X)$. The assertion concerning $\mathscr{M}(Y)$ follows by noting that

$$
\begin{aligned}
& m\left\{x \in Y: W_{t}(x) \in \mathscr{G}\right\} \\
& \quad=m\left\{x \in X: W_{t}(x) \in \mathscr{G} \cap \mathscr{M}(X)\right\}+m\left\{x \in Y \backslash X: W_{t}(x) \in \mathscr{G}\right\}
\end{aligned}
$$

for all open subsets $\mathscr{G}$ of $\mathscr{M}(Y)$, and using the above lower bounds.

Remark B.1. We explain here what improvements Theorem E brings with respect to the original version of [Kif90, Theorem 3.4].

- The latter treats the case where $P^{\tau}\left(\phi_{\mid X}\right)=0$; this follows from the relation

$$
P^{\tau}\left(\phi_{\mid X}\right)=-\lim _{\delta \rightarrow 0} \lim _{t \rightarrow+\infty} \frac{1}{t} \log a_{\delta, t}
$$

as shows (B.3), and the general assumption there which requires that for each $\delta>0$,

$$
\lim _{t \rightarrow+\infty} \frac{1}{t} \log a_{\delta, t}=0
$$

- We do not require that $\operatorname{supp} m=Y$; in fact, we only need that $m(X)>0$ in order to have the lower bounds in $\mathscr{M}(X)$, and that is ensured by the hypotheses. 
- The hypothesis in part 1 of Theorem E in order to have the upper bounds in $\mathscr{M}(Y)$ is weaker than the one of [Kif90, Theorem 3.4], where it is required that (ii) holds for all $t \in \mathfrak{T}$, all $x \in Y_{t}$ and all $\delta>0$, and that moreover (B.6) holds for all $\delta>0$.

- The hypothesis in part 2 to get the lower bounds in $\mathscr{M}(X)$ is weaker than the one of [Kif90, Theorem 3.4] since this latter requires the existence of a dense vector subspace of $C(Y)$; furthermore, these bounds are stronger than the ones in $\mathscr{M}(Y)$.

Remark B.2. For each integer $d \geq 1$ we put $\mathbb{Z}_{+}^{d}=\left\{x \in \mathbb{Z}^{d}: x_{i} \geq 0,1 \leq i \leq\right.$ $d\}$, and let $\tau$ be a continuous representation of the semi-group $\mathfrak{T} \in\left\{\mathbb{Z}_{+}^{d}, \mathbb{R}_{+}\right\}$ (resp. group $\mathfrak{T}=\mathbb{Z}^{d}$ ) in the semi-group of continuous endomorphisms (resp. group of homeomorphisms) of $X$, let $\mathscr{M}^{\tau}(X), h^{\tau}, P^{\tau}(\cdot)$ be the obvious analogues of $\mathscr{M}(X, T), h .(T), P(T, \cdot)$, respectively, and assume that $h^{\tau}$ is finite and upper semi-continuous (when $\mathfrak{T}$ is continuous, $h^{\tau}$ and $P^{\tau}(\cdot)$ are taken as the entropy and pressure of the time-one map, respectively). Let $\left(\Omega_{\alpha}\right)_{\alpha \in \wp}$ be a net of Borel probability measures on $\mathscr{M}(X)$ (in place of $\left(\Omega_{n}\right)_{n>1}$ ), and let $\left(t_{\alpha}\right)_{\alpha \in \wp}$ be a net in $(0,+\infty)$ converging to 0 (in place of $(1 / n)_{n \geq 1}$ ). It is then straightforward to verify that the statement as well as the proof of Theorem C work verbatim with the above changes (although the proof refers to some results of [DZ98] which are stated for nets indexed by positive reals, these results remain valid for general nets). Indeed, Lemma 3.1 remains true by the variational principle relating $P^{\tau}$ y $h^{\tau}$, the others required ingredients are given by the functional equality (1.5) and the hypothesis on $\mathcal{W}$, so that we just have to change the symbols in the proof.

Remark B.3. When $Y$ is $F^{t}$-invariant for all $t \in \mathfrak{T}$, then $Y=X$ and the proof of Theorem Ereveals that condition (ii) ensures that the equality (1.5) of Theorem C holds (more exactly, of its extension given by Remark B.2); the second hypothesis of part 2 of Theorem $\mathrm{E}$ is equivalent to the hypothesis on $\mathcal{W}$ of Theorem $\mathrm{C}$. Consequently, all the conclusions of Theorem E follows from the general version of Theorem $\mathrm{C}$ given by Remark B.2.

\section{REFERENCES}

[Ara07] Vítor Araújo. Large deviations bound for semiflows over a non-uniformly expanding base. Bull. Braz. Math. Soc. (N.S.), 38(3):335-376, 2007.

[Asp04] Magnus Aspenberg. The Collet-Eckmann condition for rational functions on the Riemann sphere. PhD thesis, 2004.

[Bal88] Paolo Baldi. Large deviations and stochastic homogenization. Ann. Mat. Pura Appl. (4), 151:161-177, 1988.

[Che52] Herman Chernoff. A measure of asymptotic efficiency for tests of a hypothesis based on the sum of observations. Ann. Math. Statistics, 23:493-507, 1952.

[Com03] Henri Comman. Criteria for large deviations. Trans. Amer. Math. Soc., 355(7):2905-2923 (electronic), 2003.

[Com07] Henri Comman. Variational form of the large deviation functional. Statist. Probab. Lett., 77(9):931-936, 2007.

[Com09] Henri Comman. Strengthened large deviations for rational maps and full shifts, with unified proof. Nonlinearity, 22(6):1413-1429, 2009. 
[Cra38] Harald Cramer. sur un nouveau theoreme-limite de la theorie des probabilites. Actualites Scientifiques et Industrielles, 736:5-23, 1938.

[Den96] Manfred Denker. Probability theory for rational maps. In Probability theory and mathematical statistics (St. Petersburg, 1993), pages 29-40. Gordon and Breach, Amsterdam, 1996.

[DF08] Romain Dujardin and Charles Favre. Distribution of rational maps with a preperiodic critical point. Amer. J. Math., 130(4):979-1032, 2008.

[Dob08] Neil Dobbs. Measures with positive lyapunov exponent and conformal measures in rational dynamics. arXiv:0804.3753v1, 2008.

[DPU96] Manfred Denker, Feliks Przytycki, and Mariusz Urbański. On the transfer operator for rational functions on the Riemann sphere. Ergodic Theory Dynam. Systems, 16(2):255-266, 1996.

[DS88] Nelson Dunford and Jacob T. Schwartz. Linear operators. Part I. Wiley Classics Library. John Wiley \& Sons Inc., New York, 1988. General theory, With the assistance of William G. Bade and Robert G. Bartle, Reprint of the 1958 original, A Wiley-Interscience Publication.

[DS08] Tien-Cuong Dinh and Nessim Sibony. Dynamics in several complex variables: endomorphisms of projective spaces and polynomial-like mapping. 2008. arXiv:0810.0811v1.

[DU91a] M. Denker and M. Urbański. Ergodic theory of equilibrium states for rational maps. Nonlinearity, 4(1):103-134, 1991.

[DU91b] Manfred Denker and Mariusz Urbański. On the existence of conformal measures. Trans. Amer. Math. Soc., 328(2):563-587, 1991.

[DZ98] Amir Dembo and Ofer Zeitouni. Large deviations techniques and applications, volume 38 of Applications of Mathematics (New York). Springer-Verlag, New York, second edition, 1998.

[El184] Richard S. Ellis. Large deviations for a general class of random vectors. Ann. Probab., 12(1):1-12, 1984.

[Ell85] Richard S. Ellis. Entropy, large deviations, and statistical mechanics, volume 271 of Grundlehren der Mathematischen Wissenschaften [Fundamental Principles of Mathematical Sciences]. Springer-Verlag, New York, 1985.

[ET76] Ivar Ekeland and Roger Temam. Convex analysis and variational problems. North-Holland Publishing Co., Amsterdam, 1976. Translated from the French, Studies in Mathematics and its Applications, Vol. 1.

[ET99] Ivar Ekeland and Roger Témam. Convex analysis and variational problems, volume 28 of Classics in Applied Mathematics. Society for Industrial and Applied Mathematics (SIAM), Philadelphia, PA, english edition, 1999. Translated from the French.

[FLM83] Alexandre Freire, Artur Lopes, and Ricardo Mañé. An invariant measure for rational maps. Bol. Soc. Brasil. Mat., 14(1):45-62, 1983.

[Gär77] Jurgen Gärtner. On large deviations from an invariant measure. Teor. Verojatnost. i Primenen., 22(1):27-42, 1977.

[Gri93] Jörg Grigull. Große Abweichungen und Fluktuationen für Gleichgewichtsmaße rationaler Abbildungen. PhD thesis, 1993.

[Gro03] Mikhail Gromov. On the entropy of holomorphic maps. Enseign. Math. (2), 49(3-4):217-235, 2003.

[GŚO0] Jacek Graczyk and Grzegorz Świątek. Harmonic measure and expansion on the boundary of the connectedness locus. Invent. Math., 142(3):605-629, 2000.

[Kif90] Yuri Kifer. Large deviations in dynamical systems and stochastic processes. Trans. Amer. Math. Soc., 321(2):505-524, 1990.

[KN92] Gerhard Keller and Tomasz Nowicki. Spectral theory, zeta functions and the distribution of periodic points for Collet-Eckmann maps. Comm. Math. Phys., 149(1):31-69, 1992. 
[Lju83] M. Ju. Ljubich. Entropy properties of rational endomorphisms of the riemann sphere. Ergodic Theory Dynam. Systems, 3(3):351-385, 1983.

[Lop90] Artur O. Lopes. Entropy and large deviation. Nonlinearity, 3(2):527-546, 1990.

[Mañ83] Ricardo Mañé. On the uniqueness of the maximizing measure for rational maps. Bol. Soc. Brasil. Mat., 14(1):27-43, 1983.

[MN08] Ian Melbourne and Matthew Nicol. Large deviations for nonuniformly hyperbolic systems. Trans. Amer. Math. Soc., 360(12):6661-6676, 2008.

[MS03] N. Makarov and S. Smirnov. On thermodynamics of rational maps. II. Nonrecurrent maps. J. London Math. Soc. (2), 67(2):417-432, 2003.

[Par69] William Parry. Entropy and generators in ergodic theory. W. A. Benjamin, Inc., New York-Amsterdam, 1969.

[Pla71] Detlef Plachky. On a theorem of G. L. Sievers. Ann. Math. Statist., 42:14421443, 1971.

[PRL07] Feliks Przytycki and Juan Rivera-Letelier. Statistical properties of topological Collet-Eckmann maps. Ann. Sci. École Norm. Sup. (4), 40(1):135-178, 2007.

[PRL08] Feliks Przytycki and Juan Rivera-Letelier. Nice inducing schemes and the thermodynamics of rational maps. 2008. arXiv:0806.4385 2 .

[PRLS03] Feliks Przytycki, Juan Rivera-Letelier, and Stanislav Smirnov. Equivalence and topological invariance of conditions for non-uniform hyperbolicity in the iteration of rational maps. Invent. Math., 151(1):29-63, 2003.

[PRLS04] Feliks Przytycki, Juan Rivera-Letelier, and Stanislav Smirnov. Equality of pressures for rational functions. Ergodic Theory Dynam. Systems, 24(3):891-914, 2004.

[Prz90] Feliks Przytycki. On the Perron-Frobenius-Ruelle operator for rational maps on the Riemann sphere and for Hölder continuous functions. Bol. Soc. Brasil. Mat. (N.S.), 20(2):95-125, 1990.

[PS75] D. Plachky and J. Steinebach. A theorem about probabilities of large deviations with an application to queuing theory. Period. Math. Hungar., 6(4):343-345, 1975.

[PS96] Mark Pollicott and Richard Sharp. Large deviations and the distribution of pre-images of rational maps. Comm. Math. Phys., 181(3):733-739, 1996.

[PS07] Mark Pollicott and Shrihari Sridharan. Large deviation results for periodic points of a rational map. J. Dyn. Syst. Geom. Theor., 5(1):69-77, 2007.

[PSY98] Mark Pollicott, Richard Sharp, and Michiko Yuri. Large deviations for maps with indifferent fixed points. Nonlinearity, 11(4):1173-1184, 1998.

[PU02] F. Przytycki and M. Urbański. Conformal Fractals - Ergodic Theory Methods. to appear in Cambridge University Press. Available on http://www.math.unt.edu/ urbanski and http://www.impan.edu.pl/ ${ }^{\sim}$ eliksp, 2002.

[RBY08] Luc Rey-Bellet and Lai-Sang Young. Large deviations in non-uniformly hyperbolic dynamical systems. Ergodic Theory Dynam. Systems, 28(2):587-612, 2008.

[Ree86] Mary Rees. Positive measure sets of ergodic rational maps. Ann. Sci. École Norm. Sup. (4), 19(3):383-407, 1986.

[Rue04] David Ruelle. Thermodynamic formalism. Cambridge Mathematical Library. Cambridge University Press, Cambridge, second edition, 2004. The mathematical structures of equilibrium statistical mechanics.

[Sch71] Helmut H. Schaefer. Topological vector spaces. Springer-Verlag, New York, 1971. Third printing corrected, Graduate Texts in Mathematics, Vol. 3.

[Sie69] Gerald L. Sievers. On the probability of large deviations and exact slopes. Ann. Math. Statist., 40:1908-1921, 1969.

[Smi00] Stanislav Smirnov. Symbolic dynamics and collet-eckmann conditions. Internat. Math. Res. Notices, (7):333-351, 2000. 
[SU03] Bernd O. Stratmann and Mariusz Urbanski. Real analyticity of topological pressure for parabolically semihyperbolic generalized polynomial-like maps. Indag. Math. (N.S.), 14(1):119-134, 2003.

[Tak84] Yoichiro Takahashi. Entropy functional (free energy) for dynamical systems and their random perturbations. 32:437-467, 1984.

[Tak87] Y. Takahashi. Asymptotic behaviours of measures of small tubes: entropy, Liapunov's exponent and large deviation. In Dynamical systems and applications (Kyoto, 1987), volume 5 of World Sci. Adv. Ser. Dynam. Systems, pages 1-21. World Sci. Publishing, Singapore, 1987.

[Wal82] Peter Walters. An introduction to ergodic theory, volume 79 of Graduate Texts in Mathematics. Springer-Verlag, New York, 1982.

[XF07] Hongqiang Xia and Xinchu Fu. Remarks on large deviation for rational maps on the Riemann sphere. Stoch. Dyn., 7(3):357-363, 2007.

[Zin96] Michel Zinsmeister. Formalisme thermodynamique et systèmes dynamiques holomorphes, volume 4 of Panoramas et Synthèses [Panoramas and Syntheses]. Société Mathématique de France, Paris, 1996.

$\dagger$ Henri Comman, Institute of Mathematics, Pontifical Catholic University of Valparaiso, Chile

E-mail address: henri.comman@ucv.cl

$\ddagger$ Juan Rivera-Letelier, Facultad de Matemáticas, Campus San Joaquín, P. Universidad Católica de Chile, Avenida Vicuña Mackenna 4860, Santiago, Chile

E-mail address: riveraletelier@mat.puc.cl 